\title{
Simulation for Viscoelastic Flow by a Finite Volume/Element Method
}

\author{
P. Wapperom and M.F. Webster* \\ Institute of Non-Newtonian Fluid Mechanics \\ Department of Computer Science \\ University of Wales, Swansea \\ Singleton Park, Swansea SA2 8PP, UK
}

\begin{abstract}
Stability of a second-order finite element/finite volume hybrid scheme is investigated on the basis of flows with increasing Weissenberg number. Finite elements are used to discretise the balances of mass and momentum. For the stress equation a finite volume method is used, based on the recent development with fluctuation distribution schemes for pure convection problems. Examples considered include a start-up channel flow, flow past a cylinder and the non-smooth 4:1 contraction flow for an Oldroyd-B fluid. A considerable gain in efficiency per time step can be obtained compared to an alternative pure finite element implementation. A distribution based on the flux terms is unstable for higher Weissenberg numbers, and this is also true for a distribution based on source terms alone. The instability is identified as being caused by the interaction of the balance equations and stress equation. A combination of distribution schemes based on flux and source terms, however, gives a considerable improvement to the hybrid FE/FV implementation. With respect to limiting Weissenberg number attenuation, the hybrid scheme is more stable than the pure finite element alternative for the smooth flow past a cylinder, but less so for the non-smooth contraction flow. The influence of additional strain-rate stabilisation techniques is also analysed and found to be beneficial.
\end{abstract}

Keywords: Hybrid finite element/finite volume, stability, flux and source distribution, OldroydB.

*Author for correspondence, m.f.webster@swansea.ac.uk 


\section{Introduction}

This study is concerned with establishing the properties of a new hybrid finite element/finite volume method $(\mathrm{FE} / \mathrm{FV})$ for solving viscoelastic flows, that was first proposed in Wapperom and Webster [1]. There the general rational behind such an approach was presented. The features of this technique combine the benefits of a time stepping Pressurecorrection/Taylor-Galerkin discretisation, for mass and momentum balance, with those of a cell-vertex finite volume scheme for differential constitutive equations. The introductory article [1] focused on a benchmark flow for the Oldroyd-B model, that displayed analytic form and permitted the development of a second-order formulation, competitive on efficiency with a Galerkin-Recovery finite element scheme that incorporates Petrov-Galerkin upwinding for stress (FE/SUPG). Such advances, within this cell-vertex finite volume sub-element approach, were made through the judicious treatment of flux, source and time terms of the constitutive equation. This introduced the concept of flux distribution (linear and non-linear versions), the procedure of upwinding for cell-vertex schemes and the association of cell contributions to nodal equations. In addition, for area integrals such as arise with source or time terms, the Median Dual Cell approach was considered. Consistency was observed to be a key aspect. This work was novel in the cell-vertex context, as the initial development for flux distribution was soley for pure advection problems, considered here under extension with source terms. Second order accuracy was also achieved by appealing to the parent finite element solution representation in evaluation of the sub-cell finite volume integrals. This proved to be an effective strategy to overcome the lower order of a finite volume representation based on vertex quantities.

The aim of the present work is to lay the foundations for establishing the stability properties of this FE/FV hybrid scheme, as we shift in consideration from smooth model flows to complex problems (not necessarily smooth). This leads naturally to addressing issues relevant to the derivation of steady solutions for nonlinear coupled differential problems, where stability is essential to attaining highly elastic solutions. In this manner we give careful consideration to a variety of stabilisation techniques. These include combinations of flux distribution and source/time term treatments, identifying such properties as linearity preservation and positivity, and their influence on convergence; various Recovery implementations demonstrating dependency to reach high Weissenberg numbers and different treatments for the constitutive equation; strain rate smoothing for momentum; and contrasting between quadratic and linear FV solution representation. Comparison with finite element counterparts may be used as a guidance, in terms of stability limits, accuracy and efficiency of computation.

The literature in this area was covered extensively in our precursor study [1]. Here we wish to draw particular note to the hybrid FE/FV paper of Sato and Richardson [2] for its similarity in philosophy, the articles by Tanner and co-workers [3], [4] with artificial stress diffusion, and the Discontinuous Galerkin and Galerkin least squares approach of Baaijens [5], [6]. The Sato and Richardson work for the 4:1 contraction differs from the present in adopting a FV approach for pressure and stress, with implicit time stepping and a cellcentred construction for stress. Flux corrected transport was applied to the advection terms of the constitutive equation (inconsistent treatment). The direction adopted by Tanner and co-workers is interesting in the present context due to its alternative stress stabilisation technique, that contrasts against those adopted here. The studies of Baaijens are useful for two reasons: this points to various alternative additional stabilisation possibilities from a Galerkin least squares approach, that may be incrementally incorporated consistently into 
the formulation; also there is the strain-rate smoothing for momentum, a technique explored in a number of guises [6]. For strain-rate stabilisation, see also Hulsen and van der Zanden [7], who state that this approach is common in simulations with integral models.

The algorithmic structure around which the present formulations are developed is one of a fractional stage time-stepping form. This incorporates a semi-implicit Taylor-Galerkin/PressureCorrection scheme for mass and momentum, with a combination of two-step Lax-Wendroff and Crank-Nicolson procedures. The choice for the stress discretisation is either a TaylorGalerkin form, with consistent Petrov-Galerkin streamline upwinding and recovery of velocity gradients, or a version of the FV cell-vertex sub-element scheme. Considering a twodimensional setting, first a structured triangular finite element mesh is constructed, with pressure nodes located at the vertices and velocity/stress components at both vertices and mid-side nodes. By connecting the mid-side nodes of each parent quadratic FE triangular element, four linear FV triangular subcells are formed. An advantage of such a choice is that no interpolation is required to recover the finite element nodal stress values, as the stress variables are located at the vertices of the FV cells (see also [8]).

The flow problems considered for an Oldroyd-B model, include a start-up channel flow, flow past a cylinder and flow through a four to one contraction. It is acknowledged that a limiting Weissenber number will normally be met for this model, once non-smooth flow situations are encountered. Nevertheless, this does provide a fertile regime of study for benchmark flows. In this regard, the three standard test problems are used to validate the various scheme implementations. Principally for each problem, we employ a direct comparison of $\mathrm{FV}$ variants against FE/SUPG under identical meshing. The start-up channel flow has an analytic solution. This is a smooth flow that may be used to investigate performance characteristics, seeking to identify the stability behaviour of a range of different FV implementations. During the time stepping procedure to steady-state numerical noise is present in the solution. It is the response of each individual implementation to this scenario that is under scrutiny here, and in particular, the success of the FV schemes in dealing with dominant source terms when fluxes tend to vanish. Armed with this knowledge we proceed to investigate two complex flows, flow past a cylinder that displays bifurcation points and steep stress boundary layers on curved walls, and flow through a four to one contraction with a sharp re-entrant corner as an example of a non-smooth flow. For such flows, we employ the superior implementations derived from the start-up channel flow study, seeking the path towards a limiting Weissenberg number and contrasting the quality of solutions gained, noteably in stress, from the FE/SUPG solutions.

\section{Theory}

\subsection{Governing equations}

The associated equations are those for viscoelastic incompressible flow, namely those governing the conservation of mass, transport of momentum and a constitutive law for stress. For the Oldroyd-B model the system may be expressed in non-dimensional form as:

$$
\begin{aligned}
\nabla \cdot \boldsymbol{u} & =0 \\
R e \frac{\partial \boldsymbol{u}}{\partial t} & =-R e \boldsymbol{u} \cdot \nabla \boldsymbol{u}-\nabla p+\nabla \cdot\left(2 \frac{\mu_{\mathrm{s}}}{\mu} \boldsymbol{d}+\boldsymbol{\tau}\right) \\
W e \frac{\partial \boldsymbol{\tau}}{\partial t} & =-W e \boldsymbol{u} \cdot \nabla \boldsymbol{\tau}+W e\left(\boldsymbol{L} \cdot \boldsymbol{\tau}+\boldsymbol{\tau} \cdot \boldsymbol{L}^{T}\right)+2 \frac{\mu_{\mathrm{e}}}{\mu} \boldsymbol{d}-\boldsymbol{\tau}
\end{aligned}
$$


where $\boldsymbol{u}$ is the fluid velocity, $p$ the hydrodynamic pressure, $\boldsymbol{\tau}$ the extra-stress tensor, $\mu_{\mathrm{s}}$ the solvent, $\mu_{\mathrm{e}}$ the polymeric and the total viscosity $\mu=\mu_{\mathrm{e}}+\mu_{\mathrm{s}}$, with $\boldsymbol{L}^{T}=\nabla \boldsymbol{u}$ the velocity gradient and $\boldsymbol{d}=\left(\boldsymbol{L}+\boldsymbol{L}^{T}\right) / 2$ the rate-of-deformation tensor.

Two non-dimensional group numbers are introduced of Reynolds and Weissenberg number, defined as

$$
R e=\frac{\rho U L}{\mu}, \quad W e=\frac{\lambda U}{L},
$$

where $\rho$ is the fluid density, $\lambda$ the fluid relaxation time, $U$ a characteristic velocity and $L$ a characteristic length scale of the flow. Here we expound the theory for the base case of Oldroyd-B, but note that this may be extended in a direct manner to other classes of differential models, such as the generalised Phan-Thien-Tanner, Giesekus or FENE models (see for example [9]).

\subsection{Numerical method}

The numerical approach follows a time-splitting semi-implicit formulation that, via a combination of temporal Taylor series expansions [10] and operator splitting of pressure-correction form [11], [12], generates a three fractional-stage structure per time step. Spatial discretisation then distinguishes between a pure finite element or a hybrid finite element/volume implementation, the departure lying within the treatment for stress. At the momentumcontinuity level, the implementation is that of a direct Taylor-Galerkin/Pressure-Correction scheme, well documented elsewhere [13]. This displays such features as two-step LaxWendroff time stepping, Crank-Nicolson treatment for pressure splitting and diffusion terms, a non-solenoidal solution field at stage one, a Poisson equation for temporal pressure difference at stage two, and a continuity correction for velocity at stage three.

Following Ref. [1], a general statement of the problem in discrete form is provided as follows:

Stage 1a

$$
\begin{aligned}
A_{u}\left(\mathcal{U}^{n+1 / 2}-\mathcal{U}^{n}\right) & =b_{u}\left(\mathcal{P}^{n}, \mathcal{U}^{n}, \mathcal{T}^{n}, \mathcal{D}^{n}\right), \\
\frac{2 W e}{\Delta t} A_{\tau}\left(\mathcal{T}^{n+1 / 2}-\mathcal{T}^{n}\right) & =b_{\tau}\left(\mathcal{U}^{n}, \mathcal{T}^{n}, \mathcal{D}^{n}\right),
\end{aligned}
$$

Stage $1 b$

$$
A_{u}\left(\mathcal{U}^{*}-\mathcal{U}^{n}\right)=b_{u}\left(\mathcal{P}^{n}, \mathcal{U}^{n}, \mathcal{U}^{n+1 / 2}, \mathcal{T}^{n+1 / 2}, \mathcal{D}^{n+1 / 2}\right),
$$

$$
\frac{2 W e}{\Delta t} A_{\tau}\left(\mathcal{T}^{n+1}-\mathcal{T}^{n}\right)=b_{\tau}\left(\mathcal{U}^{n+1 / 2}, \mathcal{T}^{n+1 / 2}, \mathcal{D}^{n+1 / 2}\right)
$$

Stage 2

$$
\frac{\Delta t}{2 R e} A_{2}\left(\mathcal{P}^{n+1}-\mathcal{P}^{n}\right)=b_{2}\left(\mathcal{U}^{*}\right),
$$

Stage 3

$$
\frac{2 R e}{\Delta t} A_{3}\left(\mathcal{U}^{n+1}-\mathcal{U}^{*}\right)=b_{3}\left(\mathcal{P}^{n}, \mathcal{P}^{n+1}\right),
$$

where the superscript ${ }^{n}$ denotes the time level, $\Delta t$ the time step and $\mathcal{U}, \mathcal{U}^{*}, \mathcal{P}, \mathcal{T}$ and $\mathcal{D}$ are the nodal velocity, non-solenoidal velocity, pressure, elastic stress and recovered velocity gradient vector respectively. The matrix $A_{2}$ is the standard stiffness matrix and $A_{3}$ the mass matrix. The velocity matrix $A_{u}$ is given by

$$
A_{u}=\frac{R e}{\Delta t^{*}} M+\frac{\mu_{\mathrm{s}}}{\mu} S
$$

where $\Delta t^{*}$ is the actual time step, either $\Delta t / 2$ or $\Delta t$ and the last term on the right-hand side arises via the semi-implicit discretisation of the diffusion term. This implicitness enhances 
stability, as discussed in [12]. The matrix $A_{\tau}$ equates to the identity matrix for the finite volume method, whilst for the finite element implementation would assume a mass matrix form. The exact discretisation for both $A_{\tau}$ and right-hand side $b_{\tau}$ are discussed in section 2.3. The right-hand-side vector $b_{u}$, represented in its individual terms, is given by

$$
b_{u}^{n^{\prime}}(\mathcal{P}, \mathcal{U}, \mathcal{T}, \mathcal{D})=L^{T} \mathcal{P}^{n}-\frac{\mu_{\mathrm{s}}}{\mu} S \mathcal{U}^{n}-\left(\operatorname{ReN}\left(\mathcal{U}^{n^{\prime}}\right)+\frac{\mu_{\mathrm{r}}}{\mu} S\right) \mathcal{U}^{n^{\prime}}-D\left(\mathcal{T}^{n^{\prime}}-\frac{\mu_{\mathrm{r}}}{\mu} \mathcal{D}_{\text {rec }}^{n^{\prime}}\right)+F^{n^{\prime}}
$$

where the superscript $n^{\prime}$ denotes time level $n$ in stage 1 a and level $n+1 / 2$ in stage $1 \mathrm{~b}$. The matrix $L$ is the incompressibility matrix, $N$ the convection matrix, $S$ the diffusion matrix, $D$ the elastic stress matrix and $F$ a vector containing contributions of natural boundary conditions. The term $-2 \mu_{\mathrm{r}}\left(S \mathcal{U}-D \mathcal{D}_{\text {rec }}\right) / \mu$, containing the difference of the discontinuous and the recovered continuous velocity gradient, is a strain-rate smoother. Strain rate smoothing has also been used by [7] and [6]. For $\mu_{\mathrm{r}}$ we will take $\mu_{\mathrm{r}}=\alpha \mu_{\mathrm{e}}$. With an appropriate choice of $\alpha$, stability may be increased further, as we demonstrate below.

On the temporal increment of pressure, homogeneous Neumann boundary conditions are imposed, and for $\mathcal{U}^{*}$ the same boundary conditions are imposed as to $\mathcal{U}^{n+1}$, see [11] and [12] for details. The pressure is fixed at the outflow to specify the constant of integration.

The momentum equation in stage one, and the pressure correction parts at stages two and three, are discretised via a Galerkin finite element method. The solution representation for velocity is performed with quadratic shape functions per triangular finite element, using vertices and mid-side nodes. The pressure is approximated by a linear function using the vertices alone. The extra stress, within the momentum equation, is approximated in finite element form as for velocity. As a result we obtain matrix-vector equations, which can be solved by a direct or iterative method. For reasons of accuracy, the equation for pressure in stage 2 is solved by a direct solution method, using Choleski decomposition. The momentum equations in stage one, and the incompressibility correction equation in stage three, are solved with a Jacobi iterative method using no more than five iterative sweeps [12]. In the finite volume method, the stress can be obtained directly, as we outline in section 2.5. This is in contrast to the purely finite element scheme that we use to benchmark the hybrid finite element/finite volume scheme. The purely finite element version of the TaylorGalerkin/pressure-correction method employs SUPG for the stress equations, see [14] or [1]. Furthermore, to enhance stability, recovered velocity gradients are used in this FE/SUPG method as discussed in [15]. Using the hybrid FE/FV approach avoids the need to solve a matrix-vector equation for the extra stress, and the right-hand-side vector is more straightforward to construct. This is advantageous from an efficiency viewpoint, particularly for 3D or multi-mode computations. To determine steady-state solutions, the truncation criteria for the time stepping procedure is scaled on the time step as outlined in Ref. [1], being typically $\mathcal{O}\left(10^{-m}\right)$, where $\mathrm{m}$ is greater than or equal to three.

\subsection{Finite Volume sub-cell scheme for stress}

In two dimensions, the finite element discretisation is based on piecewise continuous quadratic interpolation on triangles for velocity and stress, and linear for pressure. Solution nodal values are located at the vertices and mid-side nodes for velocity and stress, and vertices for the pressure. To apply a flux distribution scheme, we require triangles with only vertices. A cell-vertex finite volume scheme can be constructed by considering a hierarchical triangular subdivision of each parent finite element into four finite-volume subcells, as indicated 
in Fig. 1. A flux distribution scheme may then be devised, providing the updates to the individual equations associated with each vertex.

Adopting the notation of [1], the source $\boldsymbol{Q}$, flux $\mathcal{R}$ and time terms of the Oldroyd-B model may be expressed in conservative form as,

$$
\begin{aligned}
\frac{\partial \boldsymbol{\tau}}{\partial t} & =-\nabla \cdot \mathcal{R}+\boldsymbol{Q} \\
\mathcal{R} & =\boldsymbol{u} \boldsymbol{\tau} \\
\boldsymbol{Q} & =\frac{1}{W e}\left(2 \frac{\mu_{\mathrm{e}}}{\mu} \boldsymbol{d}-\boldsymbol{\tau}\right)+\boldsymbol{L} \cdot \boldsymbol{\tau}+\boldsymbol{\tau} \cdot \boldsymbol{L}^{T} .
\end{aligned}
$$

Taking each scalar stress components, $\tau$, acting in an arbitrary volume $\Omega$, its variation is controlled through the variation of the flux vector $\boldsymbol{R}=\boldsymbol{u} \tau$ and the scalar source term $Q$. With the aid of the Gauss divergence theorem on the flux term, integration of Eq. (8) over a control volume $\Omega$ for each stress component $\tau$ yields

$$
\frac{\partial}{\partial t} \int_{\Omega} \tau \mathrm{d} \Omega=\oint_{\Gamma} \boldsymbol{R} \cdot \boldsymbol{n} \mathrm{d} \Gamma+\int_{\Omega} Q \mathrm{~d} \Omega
$$

For linear FV-subcells, a linear representation (FVL) employs only its three vertex nodal values. Alternatively, as the FV-mesh is constructed directly from the parent FE-mesh (with quadratic functions), we may still retain the quadratic functions for evaluation of the FV integrals (FVQ). Clearly, this will demand more computational effort than the linear representation. However, as we have demonstrated in [1], an FVQ approach retains the second-order accuracy of the method, that would otherwise be degraded using FVL instead. For evaluation of $\boldsymbol{d}$ and $\boldsymbol{L}$, we have the following alternative choices available:

1. Based on velocity per FV subcell; constant velocity gradients per FV subcell.

2. Based on velocity over parent FE; linear velocity gradients per FV subcell, discontinuous over FE boundaries.

3. Recovered velocity gradients over surrounding finite elements; linear velocity gradients per FV subcell, continuous over FE boundaries.

The use of recovered velocity gradients is found to considerably enhance stability for the FE/SUPG implementation, see [15].

\subsection{Distribution of fluxes}

In this section, we discuss some specific choices for the distribution of flux integrals on the control volume triangle $T$ over its nodes $\{i, j, k\}$. The coefficients $\alpha_{l}$ indicate the fraction of the integral sent to node $l$. To fulfil conservation, $\alpha_{l}$ must sum to unity. For flux distribution schemes, it is important to distinguish between instances of triangles with one and two inflow sides. Both situations are illustrated in Fig. 2. The inflow sides are determined by the sign of the coefficients $k_{l}=\boldsymbol{a} \cdot \boldsymbol{n}_{l} / 2$, where $\boldsymbol{a}$ is the advection speed, that is the averaged velocity in a FV-cell, and $\boldsymbol{n}_{l}$ the inward normal on the side opposite to vertex $l$. A positive $k_{l}$ indicates that the constant advection speed vector $\boldsymbol{a}$ is inflowing across the side opposite vertex $l$. Due to the property, $\boldsymbol{n}_{i}+\boldsymbol{n}_{j}+\boldsymbol{n}_{k}=0$, it is ensured that each triangle has a maximum of two inflow and two outflow sides. 
By sending the flux of the FV-cell to the downstream node, triangular cells with only one inflow side can satisfy both the positivity and linearity preservation properties simultaneously, see [16]. For example, in the case with $k_{i}>0, k_{j}<0, k_{k}<0$, see Fig. 2, we would have

$$
\alpha_{i}=1, \quad \alpha_{j}=0, \quad \alpha_{k}=0 .
$$

The various flux distribution schemes only differ for the case of two inflow sides, as illustrated in Fig. 2, for which the flux is distributed over the nodes $i$ and $j\left(\alpha_{k}=0\right)$. We deliberate here to discuss briefly two specific flux distribution schemes that we shall consider. A linear LDB-scheme, that satisfies linearity preservation, and the PSI-scheme, a nonlinear scheme that is both positive and linearity preserving. It is noted in passing, that under general conditions, a scheme must be nonlinear to satisfy both such requirements. See [16] for an extensive discussion of these properties and other flux distribution schemes.

\subsubsection{Low Diffusion B scheme}

The Low Diffusion B (LDB) scheme is a linear $\alpha$-scheme that is linearity preserving, which demonstrates a relatively small amount of numerical diffusion in comparison with a linear positive scheme. Based on our previous experience for model problems [1], this LDB-scheme was found to be superior on accuracy to alternatives that satisfied positivity instead. The LDB-scheme is based on the angles in the triangle on both sides of the advection speed vector $\boldsymbol{a}$. With the angles $\gamma_{1}$ and $\gamma_{2}$, defined in Fig. 2, the coefficients $\alpha_{l}$ are

$$
\begin{aligned}
& \alpha_{i}=\left(\sin \gamma_{1} \cos \gamma_{2}\right) / \sin \left(\gamma_{1}+\gamma_{2}\right), \\
& \alpha_{j}=\left(\sin \gamma_{2} \cos \gamma_{1}\right) / \sin \left(\gamma_{1}+\gamma_{2}\right), \\
& \alpha_{k}=0
\end{aligned}
$$

The closer the advection speed $\boldsymbol{a}$ is to being parallel to one of the boundary sides, the larger is the contribution to the downstream node at that boundary.

\subsubsection{PSI-scheme}

The PSI-scheme is a non-linear scheme that is both positive and linearity preserving. Such a variant permits us to identify the benefits of additionally satisfying positivity. With definitions $\beta_{i}=-k_{i}\left(\tau_{i}-\tau_{k}\right)$ and $\beta_{j}=-k_{j}\left(\tau_{j}-\tau_{k}\right)$, we have for $\beta_{i}+\beta_{j}>0$,

$$
\alpha_{i}=\beta_{i} /\left(\beta_{i}+\beta_{j}\right), \quad \alpha_{j}=\beta_{j} /\left(\beta_{i}+\beta_{j}\right),
$$

and for $\beta_{i}+\beta_{j}<0$,

$$
\begin{array}{lll}
\alpha_{i}=1, & \alpha_{j}=0, & \text { for }\left|\beta_{i}\right|>\left|\beta_{j}\right|, \\
\alpha_{i}=0, & \alpha_{j}=1, & \text { for }\left|\beta_{j}\right|>\left|\beta_{i}\right| .
\end{array}
$$

For $\beta_{j}=-\beta_{i}$ (zero flux), the default setting of $\alpha_{i}=\alpha_{j}=1 / 2$ prevails.

\subsection{Distribution of sources}

A natural choice of control volume for the sources would be the median dual cell (MDC), shown in Fig. 3 for node $i$. This zone is constructed around a node on its unique control volume, by connecting midside positions to triangle centroids, and has area one third of the complete surrounding cell volume. In the original method of [16], the MDC approach has also been used for the distribution of time-derivative terms with pure convection flows. As 
the source terms are of a similar form, it would seem appropriate to treat these in a likewise fashion, by recourse to a MDC approach. However, this approach is inconsistent: there is incompatibility due to the selection of different areas for the source and flux terms, see [1]. It is our experience that, source and flux terms must be treated in a more consistent manner. Accuracy dictates the same distribution scheme should be used for both terms (recall consistent upwinding in FE).

The above deliberations lead to the following generalized form of finite volume distribution schemes in the presence of source terms,

$$
\hat{\Omega}_{l} \frac{\tau_{l}^{n+1}-\tau_{l}^{n}}{\Delta t}=\delta_{T} \alpha_{l}^{T}\left(R_{T}+Q_{T}\right)+\delta_{\mathrm{MDC}}\left(R_{\mathrm{MDC}}^{l}+Q_{\mathrm{MDC}}^{l}\right),
$$

where $R_{T}$ and $Q_{T}$ are the flux and source integral over triangle $T$, and $R_{\mathrm{MDC}}^{l}$ and $Q_{\mathrm{MDC}}^{l}$ are the flux $(\boldsymbol{u} \cdot \nabla \tau)$ and the source associated with node $l$ taken over the area of the surrounding MDC, respectively. Integration of the flux and source terms on triangle $T$ is performed by an integration rule with appropriate accuracy.

We will consider the following possibilities for the coefficients $\delta$ in Eq. (16):

I $\delta_{T}=1, \delta_{\mathrm{MDC}}=0$ : pure convection approach; anticipated to be appropriate for flux dominated flows.

II $\delta_{T}=1, \delta_{\mathrm{MDC}}=\Omega_{T} / \Omega_{\mathrm{MDC}}, Q_{T}=0, R_{\mathrm{MDC}}=0$ : (inconsistent) finite volume upwinding; the multiplication factor with the areas is needed to avoid inconsistency due to the different areas over which the flux and source terms are evaluated, leaving only inconsistency in different control volume calculations.

III $\delta_{T}=0, \delta_{\mathrm{MDC}}=1$ : pure MDC approach; anticipated to be appropriate for source dominated flows.

IV $\delta_{T}=f(W e), \delta_{\mathrm{MDC}}=1$ : similar to the consistent streamline upwind approach in finite elements; the function $f$ may be chosen as $f=\xi / 3$ if $|\xi| \leq 3$ and 1 otherwise. Here $\xi=W e U / h$ with $U$ the magnitude of the average velocity per $\mathrm{FV}$-cell and $h$ the square root of the area of the FV-cell.

$\mathrm{V} \delta_{T}=1, \delta_{\mathrm{MDC}}=f: f=1$ at the boundary points with zero velocity, $f=0$ elsewhere.

For integral evaluation on the MDC, we have used a central node sampling multiplied by the area, as in scheme IV. This is equivalent to taking a piecewise constant integrand representation. As an alternative, in scheme IVa, we have also employed a four point sampling rule based on the MDC-vertices of the FV-cell, signifying a higher order quadrature implementation (piecewise linear integrand representation).

The consistent treatment of the time-derivative term leads to $\hat{\Omega}_{l}=\alpha_{l}^{T} \hat{\Omega}_{T}$, with $\hat{\Omega}_{T}$ the area of control volume triangle T. For the MDC approach, where $\alpha_{l}^{T}=1 / 3, \hat{\Omega}_{l}$ is the area of the median dual cell around node $l$ in triangle $T$. The latter, which we will use for computations in sections $4-6$, is a one point sampling rule and avoids the need to solve a matrix-vector equation. 


\section{Problem specification}

As a model problem, we consider the start-up of Poiseuille flow in a rectangular channel. The geometry and the $2 \times 2$ mesh is displayed in Fig. 4. The geometry is confined by two walls at $y_{0}=0$ and $y_{1}=1$, where no-slip boundary conditions are adopted. Both velocity and elastic stress are imposed at the inlet $\left(x_{0}=0\right)$, and the velocity and pressure at the outlet. The prescribed velocity and stress at inflow and outflow are given by smooth timedependent boundary conditions. This is an identical flow geometry as to that considered in Carew et al. [17], although there, true transients were computed under a fixed pressure gradient, which permitted converged solutions for high Weissenberg numbers to be reached. In this paper, we solve the complete system of equations including the pressure, which is a much more severe test for the performance of the numerical method. The steady state solution consists of a quadratic profile for $u_{x}$ and $\tau_{x x}$, and a linear profile for pressure and shear stress. With a quadratic representation for velocity and stress, and a linear form for pressure, the solution can be computed to machine precision.

For more complex problems, we consider the flow past a cylinder in an infinite domain. The domain and mesh around the cylinder are shown in Fig. 4. The mesh corresponds to the mesh in [15], containing 2575 nodes in total and 20 elements on the cylinder surface. The upstream and downstream lengths are $20 R$ and $40 R$, where $R$ is the radius of the cylinder. To approximate the infinite domain we take the width of the domain equal to $40 R$. Boundary conditions are plug flow at the inlet, vanishing normal stress and tangential velocity at the outlet, and zero normal velocity, and vanishing tangential stress at the top surface. At the centreline symmetry conditions apply and at the cylinder wall no-slip boundary conditions are specified. Steep stress gradients near the top of the cylinder are encountered for this problem. To compare with the literature, we cite the experimental work of James and Acosta [18], Manero and Mena [19] and the numerical results of Townsend [20], and Pilate and Crochet [21].

A third problem, used to validate the present methodology for a complex non-smooth flow, is that of the 4:1 contraction with a sharp corner. The length of the inlet and outlet sections are $27.5 \mathrm{~L}$ and $49 \mathrm{~L}$, respectively, where $L$ is the downstream channel half-width. At the inlet both velocity and stress are imposed, and at the outlet the velocity and pressure. We have recourse to smooth Waters and King transient inlet/outlet boundary conditions [22] to reach the steady state solution. At the wall no-slip boundary conditions are specified and at the centreline symmetry conditions hold. Fig. 4 displays the computational grid, which corresponds to mesh M2, previously used with the FE/SUPG scheme of [15], consisting of 2427 nodes, with a minimum mesh size of $0.023 \mathrm{~L}$ in the vicinity of the corner. For this problem, comparison can be made widely against the literature, a survey of which was presented in [15]; see for example, Marchal and Crochet [23], Marchal and Crochet [8], Yoo and $\mathrm{Na}$ [24], Bassombrio et al. [25], and Evans and Walters [26].

In all calculations performed, we select the non-dimensional numbers of $\mu_{\mathrm{e}} / \mu=8 / 9$ and $\mu_{\mathrm{s}} / \mu=1 / 9$. For the start-up channel flow and the $4: 1$ contraction we take $R e=0$, whilst for the flow past a cylinder $R e=10$. To investigate the stability behaviour of the proposed hybrid FE/FV scheme we vary the Weissenberg number from a low value of $W e=0.1$ in specified increments of say 0.1 . This is continued until a limiting Weissenberg number $W e_{\text {crit }}$ is encountered, beyond which a converged steady-state is unattainable, giving rise to either transient oscillatory solutions or numerical divergence. 


\section{Results for start-up channel flow}

In all calculations a time step of $\Delta t=0.01$ is employed. Due to speed in attaining steady state solutions, convergence tolerances on the time-stepping scheme are varied from $\epsilon=10^{-m}$ with $m=6$ for $W e=0.1$ till $W e=3$, to $m=4$ for larger Weissenberg numbers.

\subsection{Comparison of FV-schemes}

The various possibilities for flux and source term treatment are outlined above in section 2.5. The generalised form of these schemes allows us to investigate a variety of combinations of treatments for source and flux terms, taking into account both flux and source distribution, with both consistent and non-consistent approaches. Five such schemes were uniquely identified.

The main points to observe with respect to the results charted in Tables 1-3 for startup channel flow and the comparison of the performance characteristics for the various FV scheme alternatives may be summarised as follows. Table 1 substantiates the results obtained without the application of strain-rate smoothing. Here, all schemes are seen to converge at the low value of $W e$ of 0.1 . The most efficient is scheme IV, with a form of consistent streamline upwinding similar to FE/SUPG. This situation gradually worsens through the scheme choices taking $\delta_{T}$ of unity in scheme V; worse still for scheme III (pure MDC) and scheme I (pure convection); and worst of all, is the performance of scheme II with inconsistent FV upwinding.

Across the schemes, the solution can be represented to machine precision, hence attaining identical steady state solutions. Scheme I only converges for $W e=0.1$ and slowly compared to scheme $\mathrm{V}$, whilst their only difference lies in the treatment for points at the wall. Hence, it would appear that (pure) flux distribution schemes have a problem with the vanishing velocity condition at the wall. Schemes $\mathrm{V}$ and IVa perform similarly, noteably as $W e$ increases. This would imply that the MDC treatment is of considerable significance zonally and is manifest particularly for this one-dimensional flow in the shear layers near the wall. Such a shortcoming may well apply in other flows.

At higher levels of We of say unity, the situation is reversed; scheme II (with inconsistent FV upwinding) is the superior choice. Also, we note that both schemes I and IV diverge, so that neither approaches of pure convection alone, nor MDC central-node sampling prove effective. At the same time, pure MDC of scheme III is uncompetitive, and the increased MDC sampling of scheme IVa renders convergence where scheme IV fails. It is noted that, up to this point, the FE/SUPG scheme displays superior converges properties up to $W e$ of two. This would be in keeping with our prior comparative observations on accuracy for model flows, linking superior accuracy attainment with improved high $W e$ stability behaviour.

\subsection{Recovery and strain rate stabilisation}

Table 2 represents the results with the additional strain rate stabilisation factor included. Clearly this strategy admits solutions at larger Weissenberg numbers than was previously possible. Here, a dash (-) in notation means no further solutions were attempted as the results were deteriorating from the optimal; an (X) implies that divergence was encountered. Globally, comparable levels of $W e$ are attainable for both FV and FE/SUPG, though larger $\alpha$ factors are necessary beyond $W e$ of four. This is only made possible by the careful adjustment of the weighting factor $\alpha$. An optimal setting remains to be gathered, yet it is clear that this parameter is dependent on the flow, mesh and We. At this point we have not persevered to reach an upper limit in each individual case, if indeed such applies. Clearly, a 
pure LDB-scheme (scheme I) would require a much larger value of $\alpha$ to reach convergence. Hence, computations were ceased at $W e=3$. In this instance, the distribution of fluxes and sources near the wall is not good, just as observed above in the results of Table 1. For scheme II, it seems to be the one-point MDC sampling that gives the problems. Invariably, the inaccuracy of one-point MDC sampling incurs more severe convergence penalties. This is where schemes IIa and IVa benefit. The consistency of scheme IVa eventually generates the preferred scheme of choice.

For this problem and standardising on scheme IVa, it is our observation that there is little difference between the implementations using the velocity gradient representation from the parent FE cell (discontinuous over FE cell boundaries, yet continuous over interior FV subcell boundaries) as opposed to a continuous recovered representation. This is certainly the case up to $W e=1$. The significant advantage of the latter choice is that strain rate smoothing may be invoked, the advantages of which are clearly apparent (see also Baaijens [6]).

\subsection{Alternative FV implementations}

First we comment on the inclusion of positivity within the nonlinear PSI-scheme in contrast to the LDB-scheme, both within the context of MDC approach IVa as the superior alternative cited above. On interrogating the results of Table 3, one concludes on the grounds of improved iteration count that PSI is a superior choice to the LDB scheme beyond $W e=$ 2. Nevertheless, the temporal convergence trends with the PSI scheme display oscillatory behaviour, whilst this is smooth for the LDB-scheme, and one must be careful in drawing immediate conclusions on this basis. We note that $\alpha=4$ for $W e=3$. A larger value of $\alpha$ usually decreases the number of time-steps, in the marginal region of convergence-divergence. So, if $\alpha=2$ does not converge and $\alpha=3$ does, there is an intermediate value for which the scheme converges, but is extremely slow. Taking $\alpha$ somewhat larger improves convergence considerably. For more complex flows, we would have more quantitative data to be able to illuminate the comparison, such as quality of solution.

Performance characteristics with increasing $W e$ for FVL and FVQ integral evaluation are charted in Table 4. For the linear representation FVL, strain rate stabilisation is found necessary to reach a solution at $W e=1$, and by $W e=2$, a solution could not be obtained even for a large value of $\alpha=9$. The superior level of accuracy afforded by the FVQ alternative clearly enhances stability. The FVL implementation lacks accuracy on such a coarse mesh and this is clearly demonstrated at in- and outflow regions.

We consider a decoupled approach to demonstrate that it is the coupling, and not the choice of stress discretisation, that restricts convergence from reaching high Weissenberg numbers solutions. Here, we solve for stress, whilst freezing the velocity and pressure fields at the analytical solution (a form of linearisation). In this manner, we indicate in Table 5, that high Weissenberg number solutions may be attained without difficulty. This data should be viewed in comparative form and is not intended to convey limitation on Weissenberg number attenuation. We provide the number of iterations for convergence of FE/SUPG and two FV schemes in Table 5. Such scheme variants are selected as representative of general behaviour. In addition, we have observed that fixing the stress at the analytical solution and solving for velocity and pressure, also provides no convergence difficulties for high Weissenberg numbers. It is apparent that it is not the FV discretisation itself that is responsible for the breakdown in convergence. Note that convergence of the finite volume scheme is much faster than with the finite element alternative. This leads to the realisation that as far as stability is concerned, it is the treatment of the coupling, rather than the form 
of distribution scheme, that is crucial.

Henceforth, FV-scheme IVa is adopted within the testing for complex flows.

\section{Results for cylinder flow}

For this more complex problem we adopt a time step of $\Delta t=0.005$ and a truncation tolerance $\epsilon=10^{-3}$. Results for $W e=0.1$ are obtained starting from vanishing initial conditions. For higher Weissenberg numbers, a solution is initiated from one of a lower Weissenberg number. A setting of $R e=10$ is taken, for which solutions are known to display vortex activity in the wake. It has not been found expedient to impose strain rate stabilisation for this problem, as the unstabilised FV results prove quite satisfactory in this predominately non-shearing type of flow. We will focus on scheme IVa, where we utilise the LDB-scheme for flux distribution.

In Fig. 5 at $W e=1$, we note almost identical patterns in all stress components between hybrid FE/FV and FE/SUPG schemes. The location of the top point of the cylinder is denoted by the cross-hatch. The FV scheme generally attains slightly lower peak stress values, with the exception of $\tau_{y y}$ at the leading bifurcation point. The steepest boundary layer is observed in the $\tau_{y y}$ component. At $W e=1.5$, as displayed in Fig. 6 for FV alone, the maximum value of $\tau_{y y}$ triples, from almost twenty to above sixty, over the instance of $W e=1$. Along with the large overshoot of $\tau_{y y}$ for $W e=1.5$, oscillations are apparent in the two remaining stress components around the cylinder. For this problem instance, the FV implementation is able to attain a slightly larger peak $W e$ value of 1.5 , compared with 1.4 for FE/SUPG. A fact that may be attributable to the lower stress values that arise for the FV implementation, see stress line plots around the cylinder in Fig. 5. For the hybrid FE/FV scheme, divergence was observed for $W e=1.6$, commencing from the result of $W e=1.5$. For the FE/SUPG scheme, divergence was encountered sooner at $W e=1.5$, when starting from $W e=1.4$ as the initial field. Replacing the LDB-scheme by the PSI-scheme (and so adding positivity to the flux distribution scheme), did not improve the convergence trends for high Weissenberg numbers. To illustrate this point, for a Weissenberg number of 1.4 when starting from $W e=1$, convergence could not be obtained whilst equivalently this was possible for the LDB implementation.

In Fig. 7, we provide the field contour plots for stress, in two normal components and the shear stress, to compare the results for FE/SUPG and hybrid FE/FV schemes at We of unity. The overall structure for the stress fields follow a largely similar pattern, with only minor differences being exposed around the cylinder. By default, some eighteen contours are plotted between the minimum and maximum values indicated. This agreement between both FV and FE implementations confirms accord with the literature. We note that the FE results correspond to those of Matallah et al. [15], that compared well against those of Townsend [20] and Pilate and Crochet [21].

Streamlines plots for increasing Weissenberg number and both schemes are given in Fig. 9 . Following Matallah et al., six contours are plotted between values of 2.5 and 0.1 in core flow, whilst seven are plotted in the vortex between 0.01 and - 0.008. We note that the size, strength and location of the downstream vortex follow the results of Matallah et al. [15]. There is no discernible difference between the FE/SUPG and hybrid FE/FV solutions. No vortex enhancement is detected with increasing elasticity and the downstream shift, away from the cylinder as one moves from Newtonian to elastic solutions, is replicated.

The results in Table 6 reflect the increment in number of time steps and time from one value of Weissenberg number to the next, in comparative form for FV and FE/SUPG 
implementations. The FV form always proves more economical across the range of $W e$ attempted. At $W e=0.1, \mathrm{FV}$ version is half the cost of the FE/SUPG alternative in time. This improvement degrades as $W e$ increases, to about 80 percent of the FE/SUPG time at $W e$ of unity, commencing from the previous $W e$ value. Timings are dominated in the FV case by the momentum portion of the computation (up to 75 percent); this is reduced to less than 40 percent for the FE case. Restricted to the stress equation only, the current implementation of the finite volume method is about five times more efficient than the FE/SUPG alternative. The expectation is for yet further improvement once optimisation has been accomplished. The main point here is that, for this smooth flow with steep gradients, the hybrid FE/FV scheme outperforms its FE/SUPG counterpart. We remark in passing that, in contrast, the $\mathrm{FE} / \mathrm{FV}$ scheme seems to encounter more difficulties in solving predominately shear flows.

Fig. 8 is included to demonstrate the trends with increasing $W e$ and the difference in solutions as limiting $W e$ values are approached. It is surprising that the FV scheme can reach a larger critical Weissenberg number, although the FE/SUPG scheme gives smoother solutions. Large $\tau_{y y}$ values are present with $\mathrm{FV}$ and the increase from $W e=1$ till 1.4 and 1.5 is a signal of pending divergence. Furthermore, we observe the following. With increasing Weissenberg number the stress levels are generally falling in $\tau_{x x}$ and $\tau_{x y}$. This is true for Weissenberg numbers higher than unity and is consistent with decreasing drag as others have observed. The wiggliness is associated with the occurence of a steep stress gradient before the cylinder and the lack of adequate mesh resolution there.

We note that the inclusion of the MDC approach to the LDB-scheme is a necessity for this type of flow. To clarify, we consider the element containing the bifurcation point downstream of the cylinder, see Fig. 4. This element has two points at the cylinder wall having zero velocity, and one point at the axis of symmetry, with zero normal velocity. In the case of a positive $u_{x}$, the bifurcation point will never be updated. This mechanism is not restricted to this point alone. Depending on the local velocity field, a number of nodes on the downstream side of the cylinder wall may suffer similarly, even though such nodes are connected to more elements. Consider, for example, the vertex on the cylinder wall just above the downstream bifurcation point, which is connected to three elements. In the case when the velocity in the $x$-direction is larger than that in $y$-direction, at the only node of the three elements in the interior of the flow domain, the node on the cylinder wall under consideration the will never be updated. This can be checked in a straightforward manner by applying the LDB-scheme to the three elements (two elements with one inflow side and one with two inflow sides). For the PSI-scheme, which mainly updates one node, we have noticed in our simulations that the bifurcation point upstream of the cylinder is only updated during the early stages of the time-stepping scheme (producing unphysical solution fields). Afterwards, the two elements connected to that point only send updates to the node within the domain interior and the other node at the cylinder wall. Including an MDC approach provides for nodal updates at the cylinder wall and so circumvents such difficulties.

\section{Results for 4:1 contraction flow}

For this non-smooth flow, we adopt a time step of $\Delta t=0.01$ and a truncation tolerance as above of $\epsilon=10^{-3}$. Results are provided for various Weissenberg numbers, using Waters and King transient boundary conditions [22], and restart fields from a lower Weissenberg number. Only the case of creeping flow is considered and timing performance is recorded in Table 7 . 
The FE/SUPG scheme reaches converged solutions for $W e=2$, whilst $\mathrm{FV}$ does less well in this respect (see below). The FV implementation at $W e=1$ takes just over half of the time for the FE/SUPG instance.

In Fig. 10, normal stress and shear stress are plotted at the axis of symmetry and along the horizontal line through the re-entrant corner containing the downstream wall. This provides a basis for solution comparison across the FE/SUPG and hybrid FE/FV schemes at $W e=1$. There is no discernible difference in the solution on the centreline. Some differences do become apparent between the solutions for the alternative discretisations, in the vicinity of the re-entrant corner and along the downstream wall. The location of the re-entrant corner is denoted by the cross-hatch coincident with the origin. For FV in contrast to FE/SUPG, there is a larger normal stress maximum in $\tau_{x x}$, and oscillation after the corner on the downstream wall is more pronounced. It is this response that is most probably the clearest indicator as to why $\mathrm{FV}$ fails sooner in $W e$ than does FE/SUPG for this flow. In shear stress, there is a larger peak value observed in the case of FE/SUPG.

Contour plots for normal stress and shear stress at $W e=1$ are displayed in Fig. 11 . This allows one to discern whatever differences there are on the field between the solutions derived from either scheme, FE/SUPG and hybrid FE/FV, at an appropriately largest Weissenberg number attained by both. It is clear, that both methods display very similar patterns in keeping with the stress line plots above. Most adjustment takes place in the re-entrant corner neighbourhood. With increments of 0.1 , the maximum Weissenberg number observed for the FE/FV scheme corresponds to 1.3.

Streamlines for $W e=1$ and the limiting Weissenberg numbers for both FE/SUPG and the hybrid FE/FV scheme are displayed in Fig. 13, following the format of Matallah et al. [15]. For FE/FV, there is no evidence of vortex enhancement, with the salient corner vortex reaching a strength of $-8 * 10^{-4}$ up to $W e=1.5$. The FE/SUPG solutions are similar prior to $W e=2$, whereupon a lip vortex appears of strength $-1.6 * 10^{-3}$. These findings are consistent with those reported in Matallah et al..

The additional consideration of strain rate stabilisation is also attempted for this problem. We note that, both this and channel start-up flows, have a dominant shear flow character. Here we have observed, as for the channel start-up flow, that calculations are somewhat sensitive to the setting of the weighting factor $\alpha$. For example, it was possible to increase the level of convergence from $W e$ of 1.3 to 1.4 , with a setting of $\alpha=0.3$. One needs to increase $\alpha$ further to 0.5 , to make the corresponding step from $W e$ of 1.4 to 1.5. Beyond this stage, convergence could not be achieved with any value of $\alpha$ in the range of 0.5 to unity. It would appear that fine tuning is demanded here; $\alpha$ too low is not sufficiently effective to damp numerical oscillations, whilst $\alpha$ too high will simply amplify the same and at an earlier point in the temporal procedure. From $W e=1.5$ onwards, no value of $\alpha$ could be found to provide convergence. The reason for this is most probably attributable to interaction with the pressure-correction scheme. What seems to be required is either to amend the pressure-correction scheme or to incorporate additional stabilisation to the stress equation (cf. Tanner [3]).

Fig. 12 shows the behaviour of the stress for high Weissenberg number flow for both FE/SUPG and FE/FV. Two conclusions can be drawn here: the non-zero value of $\alpha$ does not seem to influence the accuracy of the stress solutions, just convergence trends. The dip in the $x x$-component of the stress, along the downstream wall after the re-entrant corner, is unique to the FV solutions, and does not appear at $W e=2$ with FE/SUPG for example. 


\section{Conclusions}

To establish the stability of a cell-vertex finite volume method with flux distribution, we have employed three test flows for an Oldroyd-B fluid: smooth start-up channel flow, flow past a cylinder with steep gradients and non-smooth contraction flow with a sharp corner. With the start-up channel flow, we have been able to demonstrate that a distribution scheme based on fluxes alone is stable only for low Weissenberg numbers. Improved stability could be captured by including a distribution based on the source terms as well. Using a consistent combination of flux and source distribution schemes leads to a much more stable method that is competitive with the FE/SUPG implementation. An optimal combination of the flux and source distribution remains to be established. It is conspicuous that distributions based on fluxes alone experience severe problems near no-slip boundaries, and that including a supplement to the distribution from sources at wall nodes improves stability considerably. This is a finding that may well have universal application across general upwinding FV implementations. Including positivity in the flux distribution scheme, by means of the PSIscheme as instigated here, simply degrades convergence.

Solutions may be attained at considerably larger Weissenberg numbers via the incorporation of strain-rate stabilisation. Compared to FE/SUPG, however, the hybrid scheme demands more of this stabilisation. It must be noted that it is the coupling between the balance equations and the stress equations, and not the distribution schemes itself, that is responsible for such instabilities as demonstrated via a decoupled implementation. For fixed velocity and pressure fields, convergence with $\mathrm{FE} / \mathrm{FV}$ was much faster than for FE/SUPG.

The best choice scheme from the channel flow is found to be a consistent combination of flux and source distribution, similar to streamline upwinding. This scheme is applied to the more complex problems of flow past a cylinder and contraction flow. For the flow past a cylinder, similar solutions are obtained at $W e=1$ with both FE/SUPG and FE/FV. Near the limiting Weissenberg number, the stress solution with FE/SUPG remains smoother than for the hybrid scheme, although the $\mathrm{FE} / \mathrm{FV}$ scheme reaches a slightly larger Weissenberg number. For the non-smooth four to one contraction flow, the situation is reversed with the FE/SUPG scheme attaining a larger limiting Weissenberg number of two, compared to 1.5 for the FE/FV case. Stress profiles appear similar on the field, with differences in the solutions showing up in the re-entrant corner neighbourhood. In addition, oscillation in the normal stress along the downstream wall is more pronounced with FE/FV than FE/SUPG. This is an issue that demands more detailed attention with a view to local accuracy considerations.

The benefit of the FE/FV hybrid scheme for complex flows lies in the improvement on efficiency. Compared to the FE/SUPG alternative it requires both less memory and CPUtime. Future research will be directed towards a sytematic study of further optimisation, where both reduction in CPU-time usage and improved stability will be sought. For example, stability may be improved in a number of ways: by improving the flux and source distribution, or its combination; including stabilisation in the stress equation; or addressing the coupling of the system.

\section{Acknowledgements}

The financial support of EPSRC ROPA grant GR/K63801 and EU/TMR Network grant FMRX-CT98-0210 is gratefully acknowledged. The authors would like to thank our joint investigators on this work, Professor P. Townsend and Dr R. Gwynllyw for their contributions, and Dr K.S. Sujatha for her assistance in the final drafting stages of the manuscript. 


\section{References}

[1] P. Wapperom and M.F. Webster. A second-order hybrid finite element/volume method for viscoelastic flows. In press J. Non-Newtonian Fluid Mech.

[2] T. Sato and S.M. Richardson. Explicit numerical simulation of time-dependent viscoelastic flow problems by a finite-element/finite-volume method. J. Non-Newtonian Fluid Mech., 51:249-275, 1994.

[3] S.-C. Xue, N. Phan-Thien, and R.I. Tanner. Numerical study of secondary flows of viscoelastic fluid in straight pipes by an implicit finite volume method. J. Non-Newtonian Fluid Mech., 59:191-213, 1995.

[4] X. Huang, N. Phan-Thien, and R.I. Tanner. Viscoelastic flow between eccentric rotating cylinders: unstructured control volume method. J. Non-Newtonian Fluid Mech., 64:7192, 1996.

[5] F.P.T. Baaijens. Application of low-order discontinuous Galerkin methods to the analysis of viscoelastic flows. J. Non-Newtonian Fluid Mech., 52:37-57, 1994.

[6] F.P.T. Baaijens. An iterative solver for the DEVSS/DG method with application to smooth and non-smooth flows of upper convected Maxwell fluid. J. Non-Newtonian Fluid Mech., 75:119-138, 1998.

[7] M.A. Hulsen and J.P.P.M. van der Zanden. Numerical simulation of contraction flows using a multi-mode Giesekus model. J. Non-Newtonian Fluid Mech., 38:183-221, 1991.

[8] J.M. Marchal and M.J. Crochet. A new mixed finite element for calculating viscoelastic flow. J. Non-Newtonian Fluid Mech., 26:77-114, 1987.

[9] R.B. Bird, R.C. Armstrong, and O. Hassager. Dynamics of polymeric liquids, volume 1. John Wiley, New York, 2nd edition, 1987.

[10] J. Donea. A Taylor-Galerkin method for convective transport problems. Internat. J. Numer. Methods Engrg., 20:101-119, 1984.

[11] P. Townsend and M.F. Webster. An algorithm for the three-dimensional transient simulation of non-Newtonian fluid flows. In G.N. Pande and J. Middleton, editors, Transient/dynamic analysis and constitutive laws for engineering materials, Int. Conf. Numerical Methods in Engineering: Theory and Applications - NUMETA 87, volume 2, pages T12/1-11. Kluwer Academic Publishers, Dordrecht, 1987.

[12] D.M. Hawken, P. Townsend, and M.F. Webster. A Taylor-Galerkin-based algorithm for viscous incompressible flow. Int. J. Num. Methods Fluids, 10:327-351, 1990.

[13] A. Baloch, P. Townsend, and M.F. Webster. On two- and three-dimensional expansion flows. Computers \& Fluids, 24:863-882, 1995.

[14] E.O.A. Carew, P. Townsend, and M.F. Webster. A Taylor-Petrov-Galerkin algorithm for viscoelastic flow. J. Non-Newtonian Fluid Mech., 50:253-287, 1993.

[15] H. Matallah, P. Townsend, and M.F. Webster. Recovery and stress-splitting schemes for viscoelastic flows. J. Non-Newtonian Fluid Mech., 75:139-166, 1998. 
[16] R. Struijs, H. Deconinck, and P.L. Roe. Fluctuation splitting for the 2D Euler equations. Technical Report Lecture series 1990-01, Von Karman Institute for Fluid Dynamics, 1991.

[17] E.O. Carew, P. Townsend, and M.F. Webster. Taylor-Galerkin algorithms for viscoelastic flow: application to a model problem. Num. Meth. Partial Differential Equations, 19:171-190, 1994.

[18] D.F. James and A.J. Acosta. The laminar flow of dilute polymer solutions around circular cylinders. J. Fluid Mech., 42:269-288, 1970.

[19] O. Manero and B. Mena. On the slow flow of viscoelastic liquids past a circular cylinder. J. Non-Newtonian Fluid Mech., 9:379-387, 1981.

[20] P. Townsend. A numerical simulation of Newtonian and viscoelastic flow past stationary and rotating cylinders. J. Non-Newtonian Fluid Mech., 6:219-243, 1980.

[21] G. Pilate and M.J. Crochet. Plane flow of a second-order fluid past submerged boundaries. J. Non-Newtonian Fluid Mech., 2:323-341, 1977.

[22] N.D. Waters and M.J. King. Unsteady flow of an elastico-viscous liquid. Rheol. Acta, 9:345-355, 1970.

[23] J.M. Marchal and M.J. Crochet. Hermitian finite elements for calculating viscoelastic flow. J. Non-Newtonian Fluid Mech., 20:187-207, 1986.

[24] J.Y. Yoo and Y. Na. A numerical study of the planar contraction flow of a viscoelastic fluid using the SIMPLER algorithm. J. Non-Newtonian Fluid Mech., 39:89-106, 1991.

[25] F.G. Basombrio, G.C. Buscaglia, and E.A. Dari. Simulation of highly elastic fluid flows without additional numerical diffusivity. J. Non-Newtonian Fluid Mech., 39:189-206, 1991.

[26] R.E. Evans and K. Walters. Further remarks on the lip-vortex mechanism of vortex enhancement in planar-contraction flows. J. Non-Newtonian Fluid Mech., 32:95-105, 1989. 


\section{Table legend}

Table 1: Number of time steps for various FV-schemes and FE/SUPG, start-up channel flow.

Table 2: Number of time steps for various FV-schemes and FE/SUPG; start-up channel flow, with strain-rate stabilizing, results for $W e=4$ and higher from lower Weissenberg number initial field.

Table 3: Number of time steps for LDB- and PSI-scheme with MDC approach IVa; start-up channel flow, with strain-rate stabilizing, results for $W e=4$ from lower Weissenberg number initial field.

Table 4: Number of time steps for FVL and FVQ; LDB-scheme with MDC approach IVa, start-up channel flow.

Table 5: Number of time steps for FV and FE/SUPG for start-up channel flow; decoupled equations.

Table 6: Number of time steps $\Delta N$ and CPU-time $\Delta T$ for FE/SUPG and hybrid FE/FV, flow past a cylinder, various Weissenberg numbers, started from lower Weissenberg number initial field.

Table 7: Number of time steps $\Delta N$ and CPU-time $\Delta T$ for various $F V$-schemes and FE/SUPG, 4:1 contraction flow, result for $W e=2$ started from lower Weissenberg number initial field. 


\section{Figure legend}

Figure 1: Schematic diagram of finite element with four finite volume subcells, and variable location.

Figure 2: FV triangular cell with (a) one inflow side, (b) two inflow sides, and (c) graphical representation of LDB-scheme defining $\gamma_{1}$ and $\gamma_{2}$.

Figure 3: Triangular finite volume grid with median dual cell (MDC) for node $i$.

Figure 4: Finite element mesh for a) start-up channel flow, b) flow past a cylinder zoomed at the cylinder, and c) 4:1 contraction flow zoomed at the corner.

Figure 5: Line plot of stress at centreline and cylinder wall, flow past a cylinder; $W e=1$, FE/SUPG and hybrid FE/FV; a) $\tau_{x x}$, b) $\tau_{x y}$, c) $\tau_{y y}$.

Figure 6: Line plot of stress at centreline and cylinder wall, flow past a cylinder for $W e=1$ and $W e=1.5$, hybrid FE/FV; a) $\tau_{x x}$, b) $\tau_{x y}$, c) $\tau_{y y}$.

Figure 7: Contour lines of stress, normal and shear, flow past a cylinder, $W e=1, \mathrm{FE} / \mathrm{SUPG}$ and hybrid FE/FV, a), d) $\tau_{x x}$ : -0.72 to 25.29 , b), e) $\tau_{x y}:-0.94$ to $6.52 \mathrm{c}$ ), f) $\tau_{y y}:-0.43$ to 2.88 .

Figure 8: Line plot of stress at centreline and cylinder wall, flow past a cylinder for $W e=1$, $W e=1.4$, and $W e=1.5$, hybrid FE/FV and FE/SUPG; a) $\tau_{x x}$, b) $\tau_{x y}$, c) $\tau_{y y}$.

Figure 9: Streamlines for cylinder flow, FE/SUPG, a) $W e=1$, b) $W e=1.4$, and hybrid $\mathrm{FE} / \mathrm{FV}, \mathrm{c}) W e=1, \mathrm{~d}) W e=1.4, \mathrm{e}) W e=1.5$.

Figure 10: Line plot of stress at centre line a) $\tau_{x x}$ and $\tau_{y y}$ and horizontal line through reentrant corner b) $\tau_{x x}$ and $\tau_{y y}$, c) $\tau_{x y}$; 4:1 contraction flow, FE/SUPG and hybrid FE/FV, $W e=1$.

Figure 11: Contour lines of stress, normal and shear, 4:1 contraction flow, $W e=1$, FE/SUPG and hybrid FE/FV a), d) $\tau_{x x}:-0.51$ to 9.21, b), e) $\tau_{x y}:-0.04$ to 9.25 c), f) $\tau_{y y}:-0.31$ to 17.85 .

Figure 12: Line plot of stress at horizontal line through re-entrant corner a) $\tau_{x x}$, b) $\tau_{x y}$, and c) $\tau_{y y} ; 4: 1$ contraction flow, FE/SUPG and hybrid FE/FV, various Weissenberg numbers.

Figure 13: Streamlines for 4:1 contraction flow, FE/SUPG, a) $W e=1$, b) $W e=2$, and hybrid FE/FV, c) $W e=1$, d) $W e=1.3$, e) $W e=1.5$. 
Table 1: Number of time steps for various FV-schemes and FE/SUPG, start-up channel flow.

\begin{tabular}{c|r|c|c|c|c|c|c}
\hline & \multicolumn{1}{|c|}{ FE } & \multicolumn{6}{|c}{$\mathrm{FV}$} \\
\hline$W e$ & SUPG & I & II & III & IV & IVa & V \\
\hline 0.1 & 291 & 419 & 1114 & 360 & 177 & 290 & 289 \\
1.0 & 2058 & $\mathrm{X}$ & 2278 & 22080 & $\mathrm{X}$ & 7288 & 7976 \\
2.0 & 5672 & & $\mathrm{X}$ & $\mathrm{X}$ & & $\mathrm{X}$ & $\mathrm{X}$ \\
3.0 & $\mathrm{X}$ & & & & & & \\
\hline
\end{tabular}

Table 2: Number of time steps for various FV-schemes and FE/SUPG; start-up channel flow, with strain-rate stabilizing, results for $W e=4$ and higher from lower Weissenberg number initial field.

\begin{tabular}{c|c|c|c|c|c|c|c|c|c|c}
\hline & \multicolumn{2}{|c|}{ FE } & \multicolumn{10}{c}{ FV } \\
\hline$W e$ & $\alpha$ & SUPG & $\alpha$ & I & $\alpha$ & II & $\alpha$ & IIa & $\alpha$ & IVa \\
\hline 0.1 & 0 & 291 & 0 & 419 & 0 & 1114 & - & 1128 & 0 & 290 \\
1.0 & 0 & 2058 & 1 & 3624 & 0 & 2278 & - & 2418 & 0 & 7288 \\
2.0 & 0 & 5672 & 2 & 19594 & 1 & 20761 & 1 & 9814 & 1 & 17656 \\
3.0 & 1 & 11196 & 7 & 18454 & 7 & X & 4 & 19509 & 3 & 14981 \\
4.0 & 1 & 18745 & - & - & & & 7 & 17040 & 7 & 10111 \\
5.0 & 3 & 27968 & - & - & & & - & - & 12 & 23055 \\
\hline
\end{tabular}

Table 3: Number of time steps for LDB- and PSI-scheme with MDC approach IVa; start-up channel flow, with strain-rate stabilizing, results for $W e=4$ from lower Weissenberg number initial field.

\begin{tabular}{c|c|c|c|c}
\hline$W e$ & $\alpha$ & PSI & $\alpha$ & LDB \\
\hline 0.1 & 0 & 290 & 0 & 290 \\
1.0 & 0 & 6691 & 0 & 7288 \\
2.0 & 1 & 18049 & 1 & 17656 \\
3.0 & 4 & 10624 & 3 & 14981 \\
4.0 & 7 & 6613 & 7 & 10111 \\
\hline
\end{tabular}

Table 4: Number of time steps for FVL and FVQ; LDB-scheme with MDC approach IVa, start-up channel flow.

\begin{tabular}{c|c|c|c|c}
\hline$W e$ & $\alpha$ & FVL & $\alpha$ & FVQ \\
\hline 0.1 & 0 & 285 & 0 & 290 \\
1.0 & 1 & 19453 & 0 & 7288 \\
2.0 & & $\mathrm{X}$ & 1 & 17656 \\
\hline
\end{tabular}


Table 5: Number of time steps for FV and FE/SUPG for start-up channel flow; decoupled equations.

\begin{tabular}{c|c|c|c}
\hline$W e$ & FE/SUPG & FV-I & FV-IVa \\
\hline 10 & 10005 & 8735 & 4683 \\
100 & 13951 & 10385 & 5659 \\
500 & 15210 & 11076 & 5945 \\
1000 & 15662 & 11361 & 6050 \\
\hline
\end{tabular}

Table 6: Number of time steps $\Delta N$ and CPU-time $\Delta T$ for FE/SUPG and hybrid FE/FV, flow past a cylinder, various Weissenberg numbers, started from lower Weissenberg number initial field.

\begin{tabular}{c|c|c|c|c}
\hline & \multicolumn{2}{|c|}{$\mathrm{FE} / \mathrm{SUPG}$} & \multicolumn{2}{c}{$\mathrm{FV}$} \\
\hline$W e$ & $\Delta N$ & $\Delta T(\mathrm{~s})$ & $\Delta N$ & $\Delta T(\mathrm{~s})$ \\
\hline 0.1 & 1687 & 1092 & 1687 & 528 \\
0.5 & 1226 & 780 & 1029 & 315 \\
0.8 & 2214 & 1424 & 2705 & 841 \\
1.0 & 2767 & 1766 & 4479 & 1382 \\
1.5 & $\mathrm{X}$ & $\mathrm{X}$ & 6971 & 2169 \\
1.8 & & & $\mathrm{X}$ & $\mathrm{X}$ \\
\hline
\end{tabular}

Table 7: Number of time steps $\Delta N$ and CPU-time $\Delta T$ for various FV-schemes and FE/SUPG, 4:1 contraction flow, result for $W e=2$ started from lower Weissenberg number initial field.

\begin{tabular}{c|c|c|c|c}
\hline & \multicolumn{2}{|c|}{ FE/SUPG } & \multicolumn{2}{c}{$\mathrm{FV}$} \\
\hline$W e$ & $\Delta N$ & $\Delta T(\mathrm{~s})$ & $\Delta N$ & $\Delta T(\mathrm{~s})$ \\
\hline 0.1 & 328 & 260 & 338 & 162 \\
1.0 & 1396 & 1128 & 1740 & 885 \\
2.0 & 2593 & 2333 & $\mathrm{X}$ & $\mathrm{X}$ \\
\hline
\end{tabular}




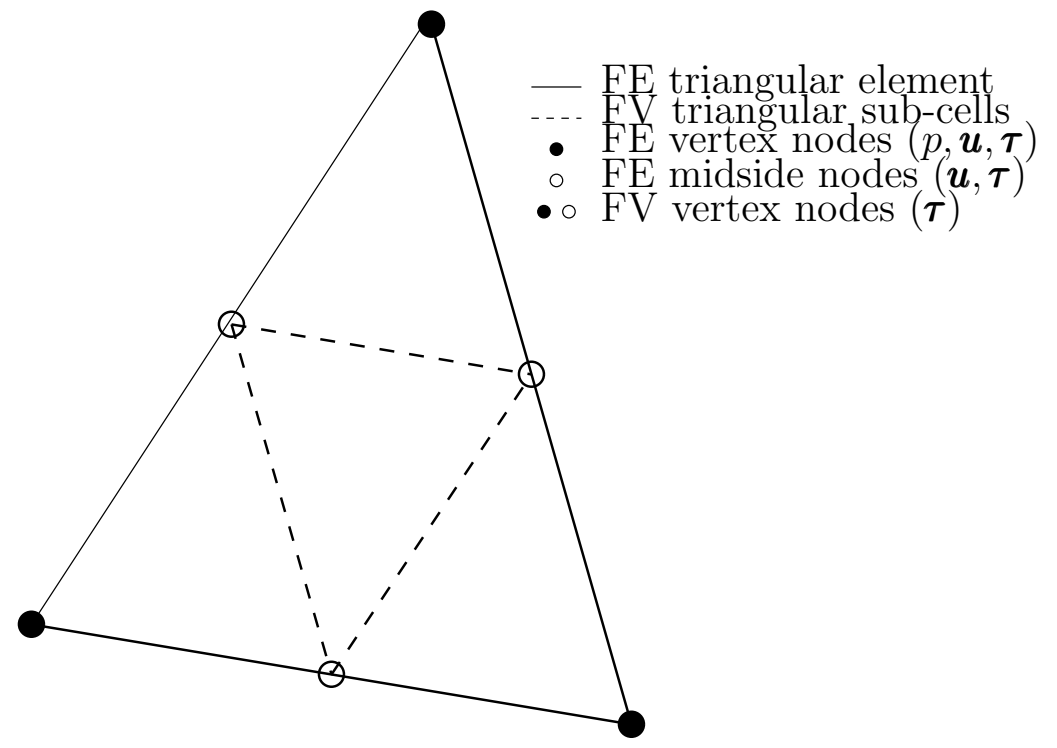

Figure 1: Schematic diagram of finite element with four finite volume subcells, and variable location. 

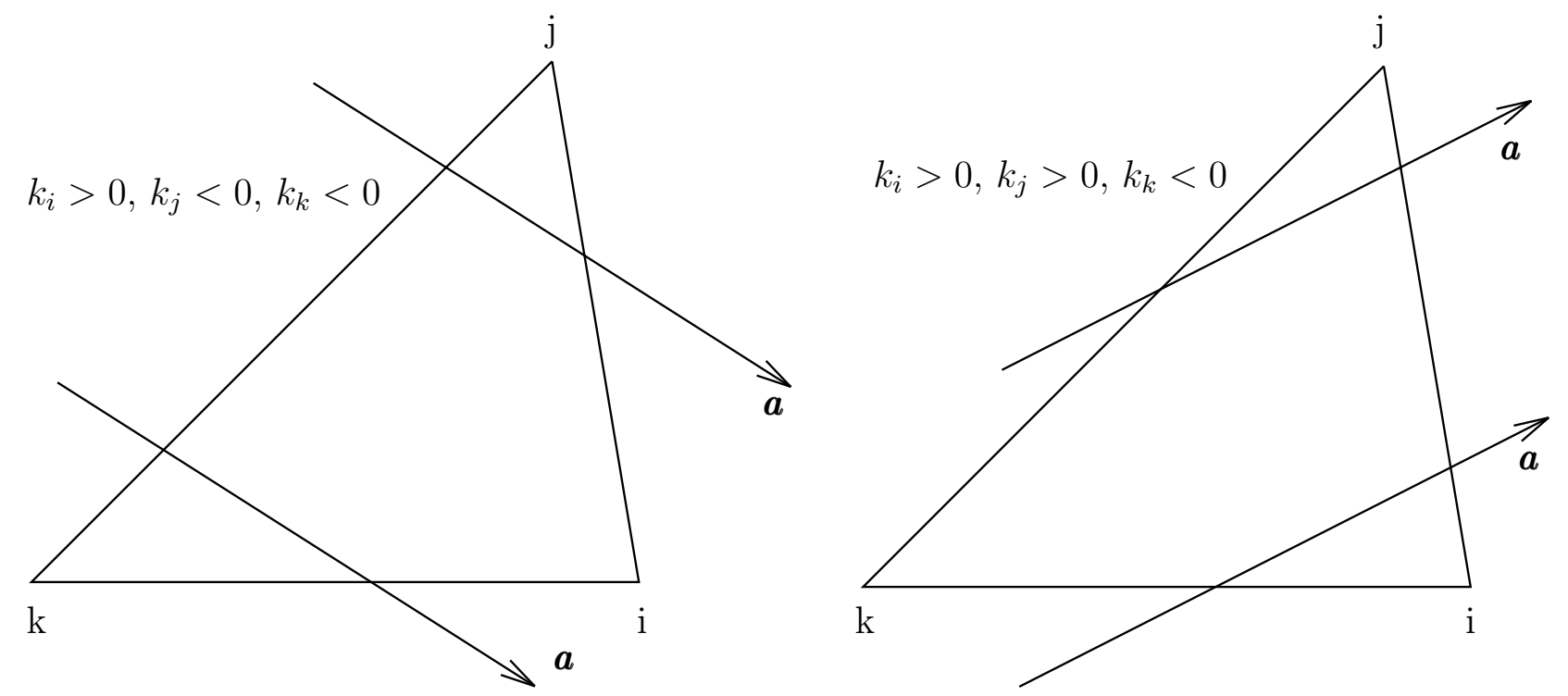

a)

b)

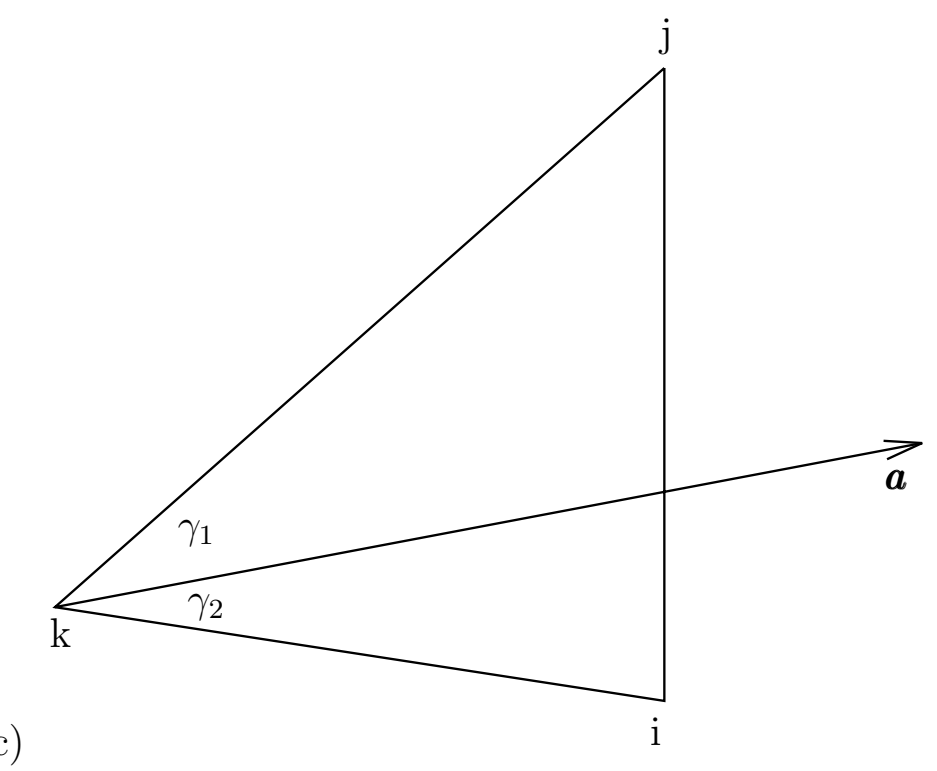

Figure 2: FV triangular cell with (a) one inflow side, (b) two inflow sides, and (c) graphical representation of LDB-scheme defining $\gamma_{1}$ and $\gamma_{2}$. 


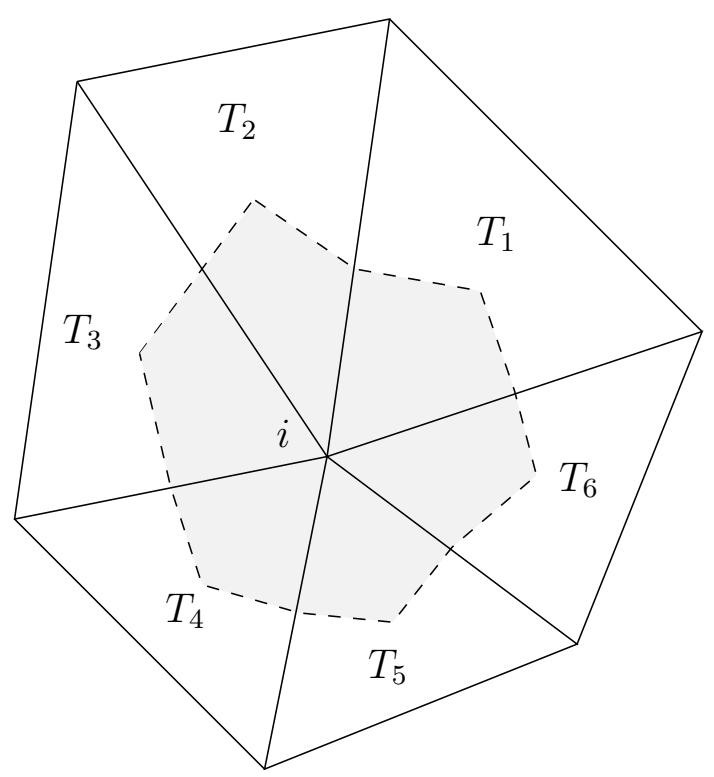

Figure 3: Triangular finite volume grid with median dual cell (MDC) for node $i$. 
a)

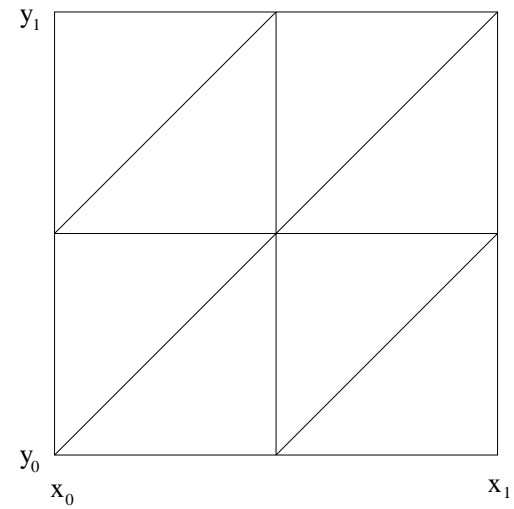

b)

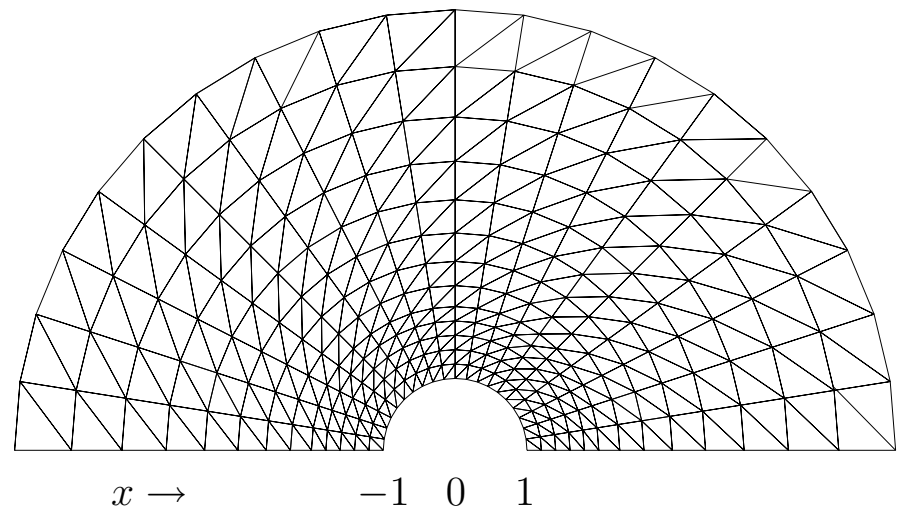

c)
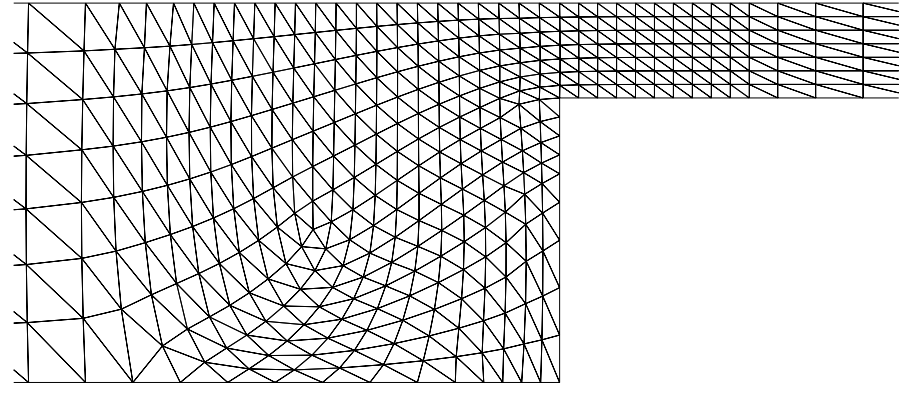
$-5.6$
$x \rightarrow$
0
5.3

Figure 4: Finite element mesh for a) start-up channel flow, b) flow past a cylinder zoomed at the cylinder, and c) 4:1 contraction flow zoomed at the corner. 

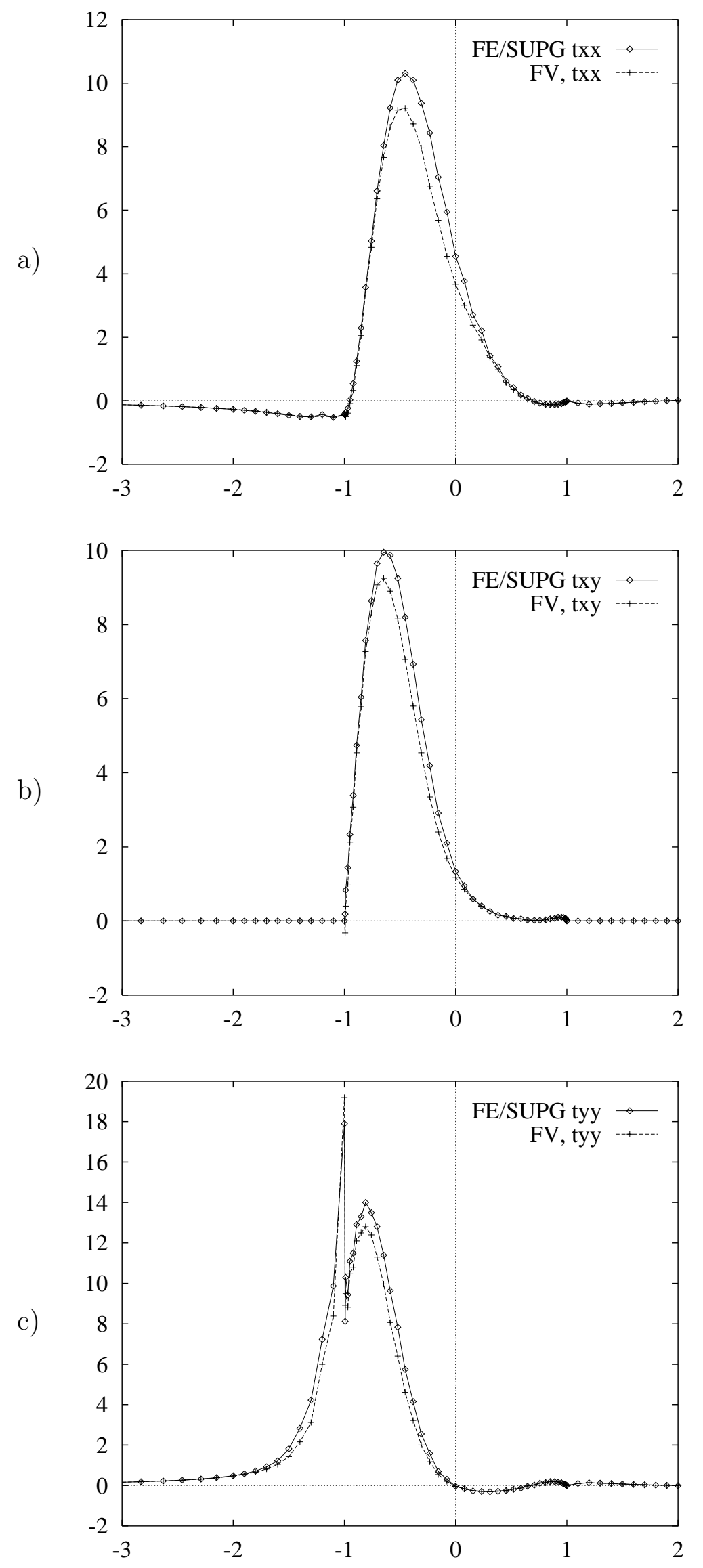

Figure 5: Line plot of stress at centreline and cylinder wall, flow past a cylinder; $W e=1$, FE/SUPG and hybrid FE/FV; a) $\tau_{x x}$, b) $\left.\tau_{x y}, \mathrm{c}\right) \tau_{y y}$. 

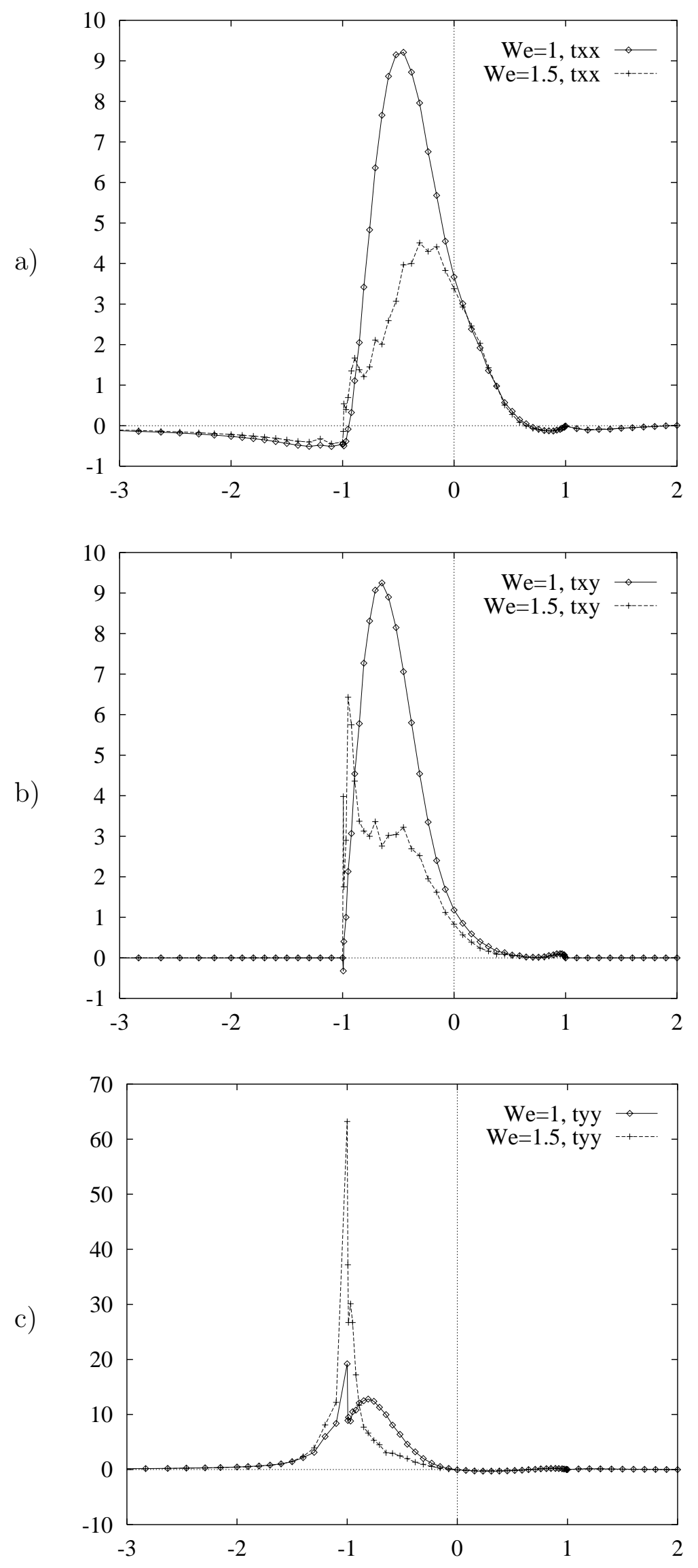

Figure 6: Line plot of stress at centreline and cylinder wall, flow past a cylinder for $W e=1$ and $W e=1.5$, hybrid FE/FV; a) $\tau_{x x}$, b) $\tau_{x y}$, c) $\tau_{y y}$. 
FE/SUPG

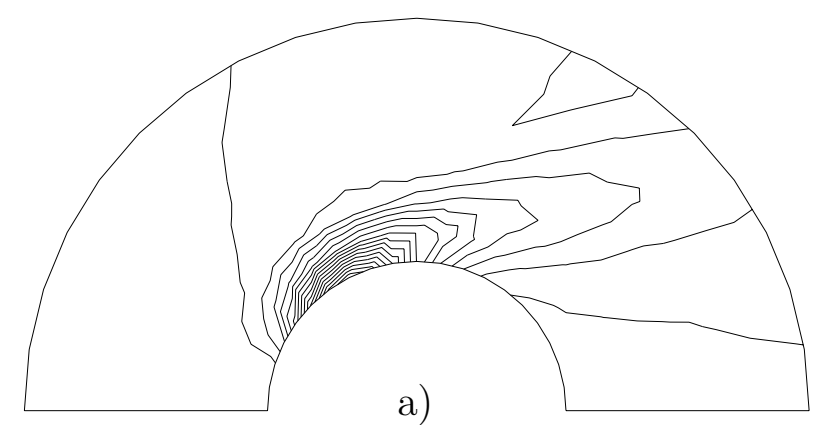

a)

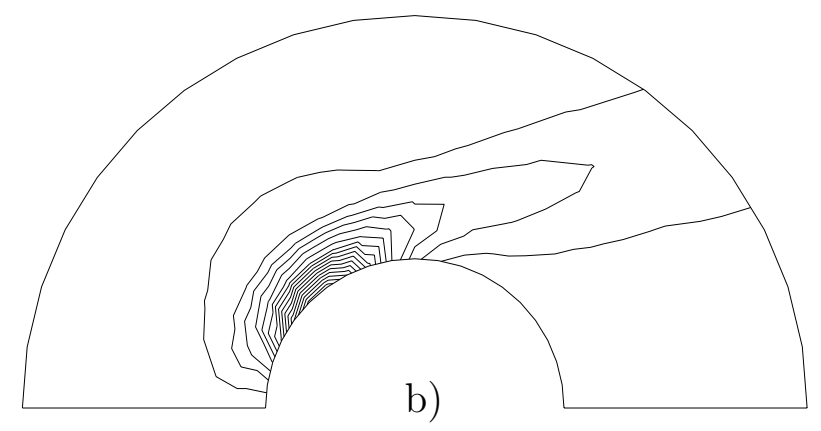

b)

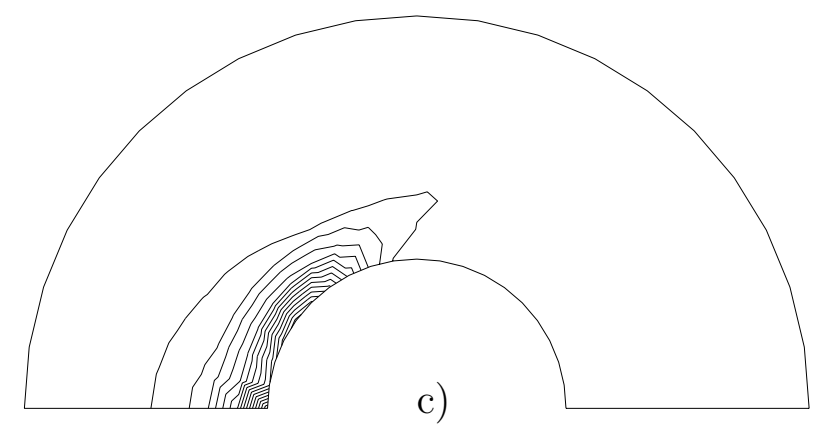

$\mathrm{FE} / \mathrm{FV}$

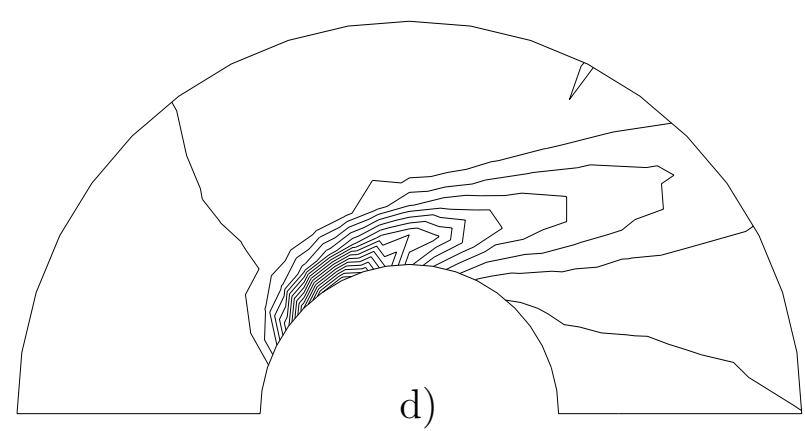

d)

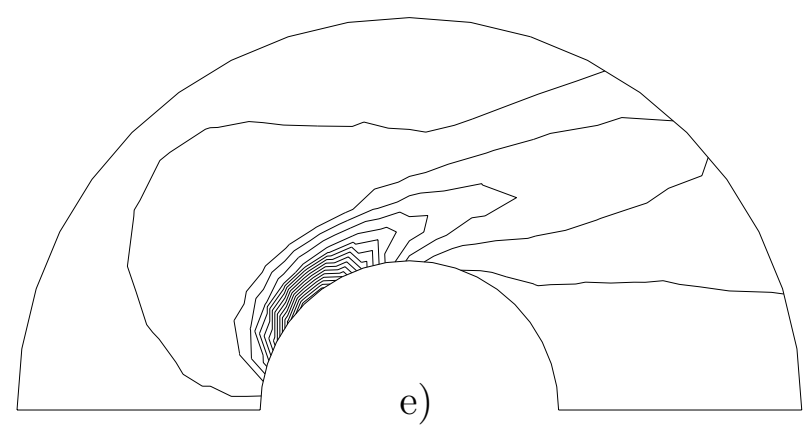

e)

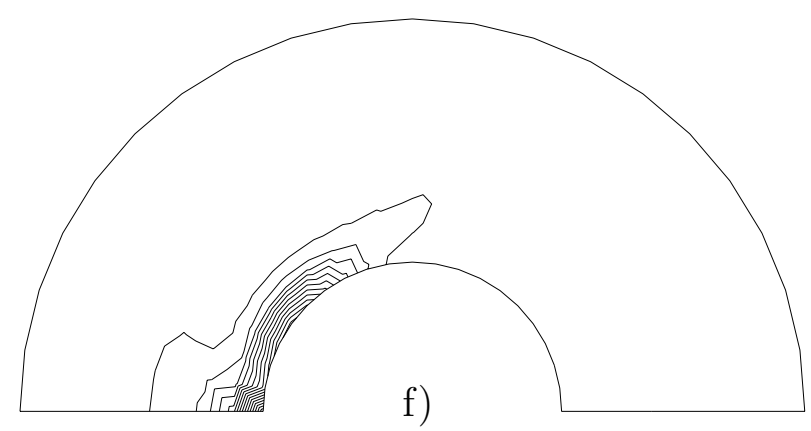

Figure 7: Contour lines of stress, normal and shear, flow past a cylinder, $W e=1, \mathrm{FE} / \mathrm{SUPG}$ and hybrid FE/FV, a), d) $\tau_{x x}:-0.72$ to 25.29 , b), e) $\tau_{x y}:-0.94$ to $6.52 \mathrm{c}$ ), f) $\tau_{y y}:-0.43$ to 2.88 . 

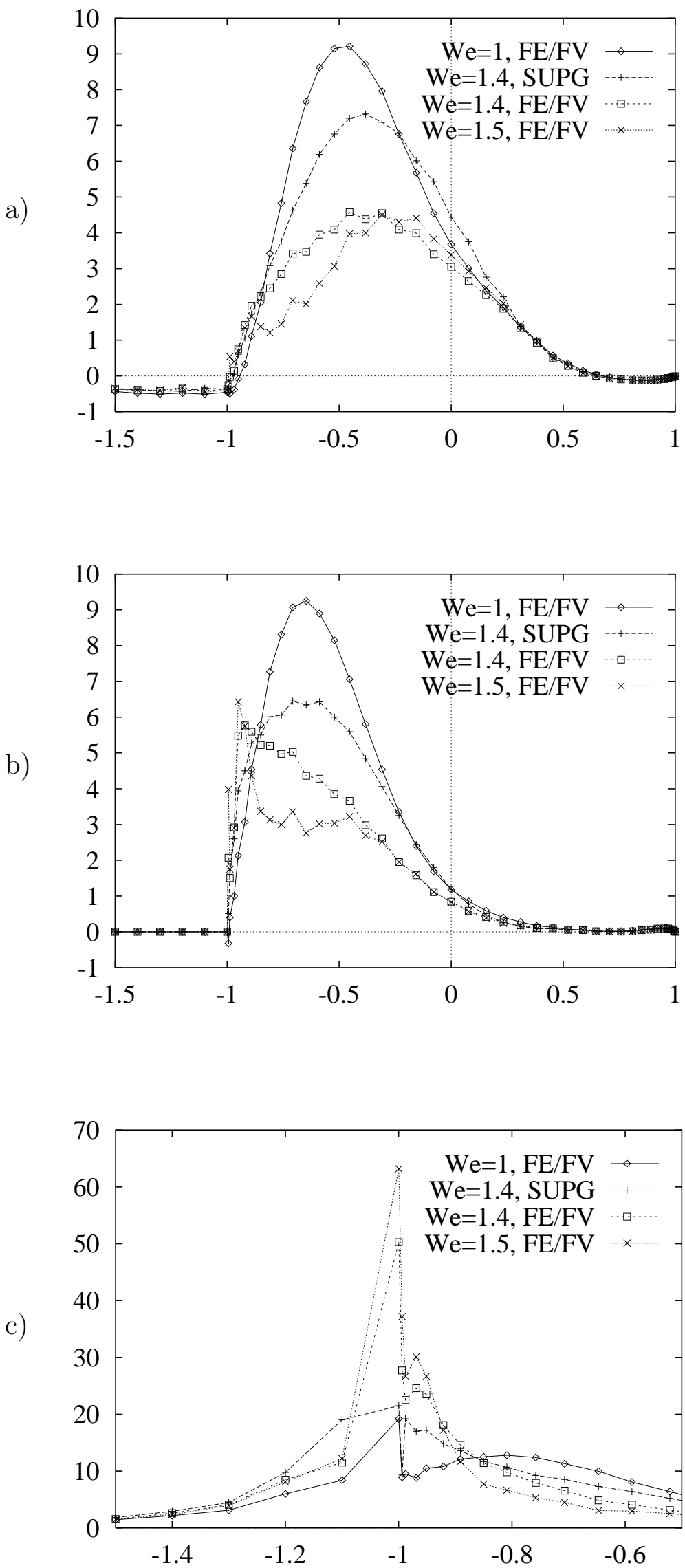

Figure 8: Line plot of stress at centreline and cylinder wall, flow past a cylinder for $W e=1$, $W e=1.4$, and $W e=1.5$, hybrid FE/FV and FE/SUPG; a) $\tau_{x x}$, b) $\tau_{x y}$, c) $\tau_{y y}$. 
FE/SUPG

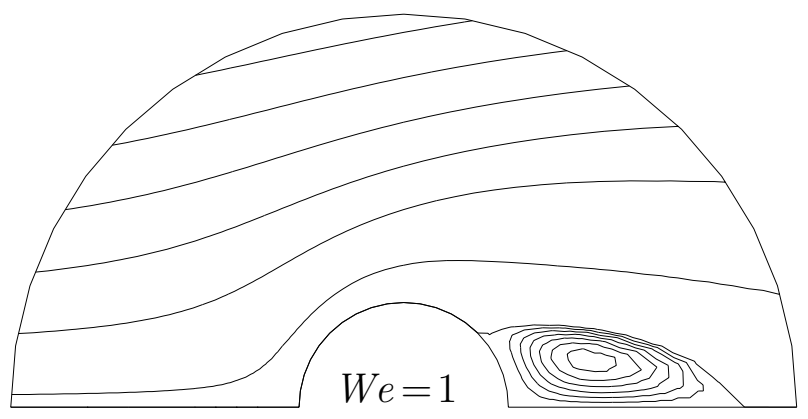

a)

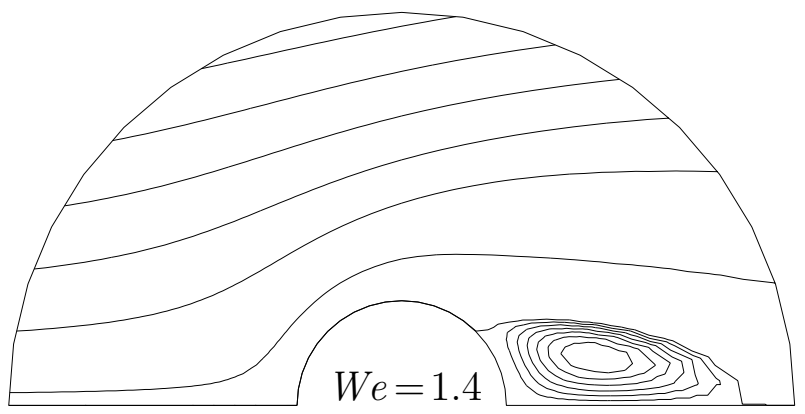

b)
$\mathrm{FE} / \mathrm{FV}$

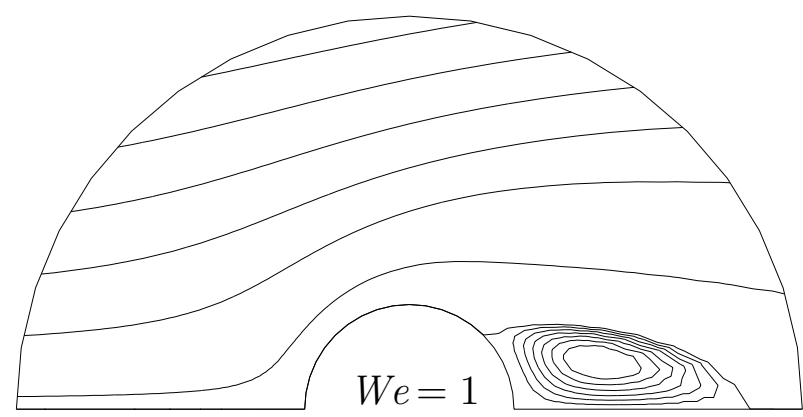

c)

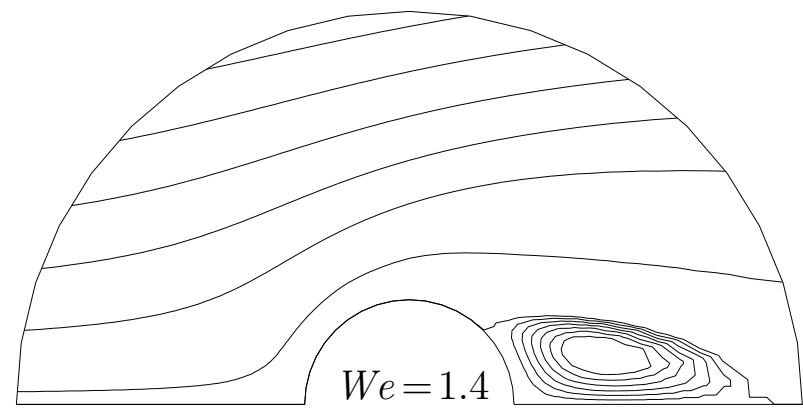

d)

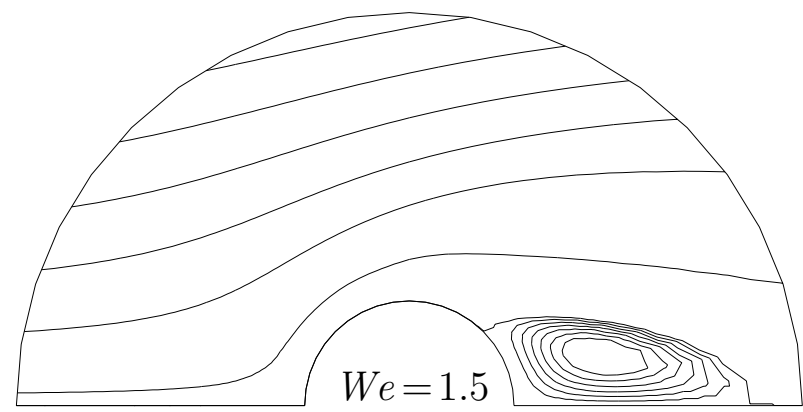

e)

Figure 9: Streamlines for cylinder flow, FE/SUPG, a) $W e=1$, b) $W e=1.4$, and hybrid $\mathrm{FE} / \mathrm{FV}, \mathrm{c}) W e=1, \mathrm{~d}) W e=1.4, \mathrm{e}) W e=1.5$. 
a)
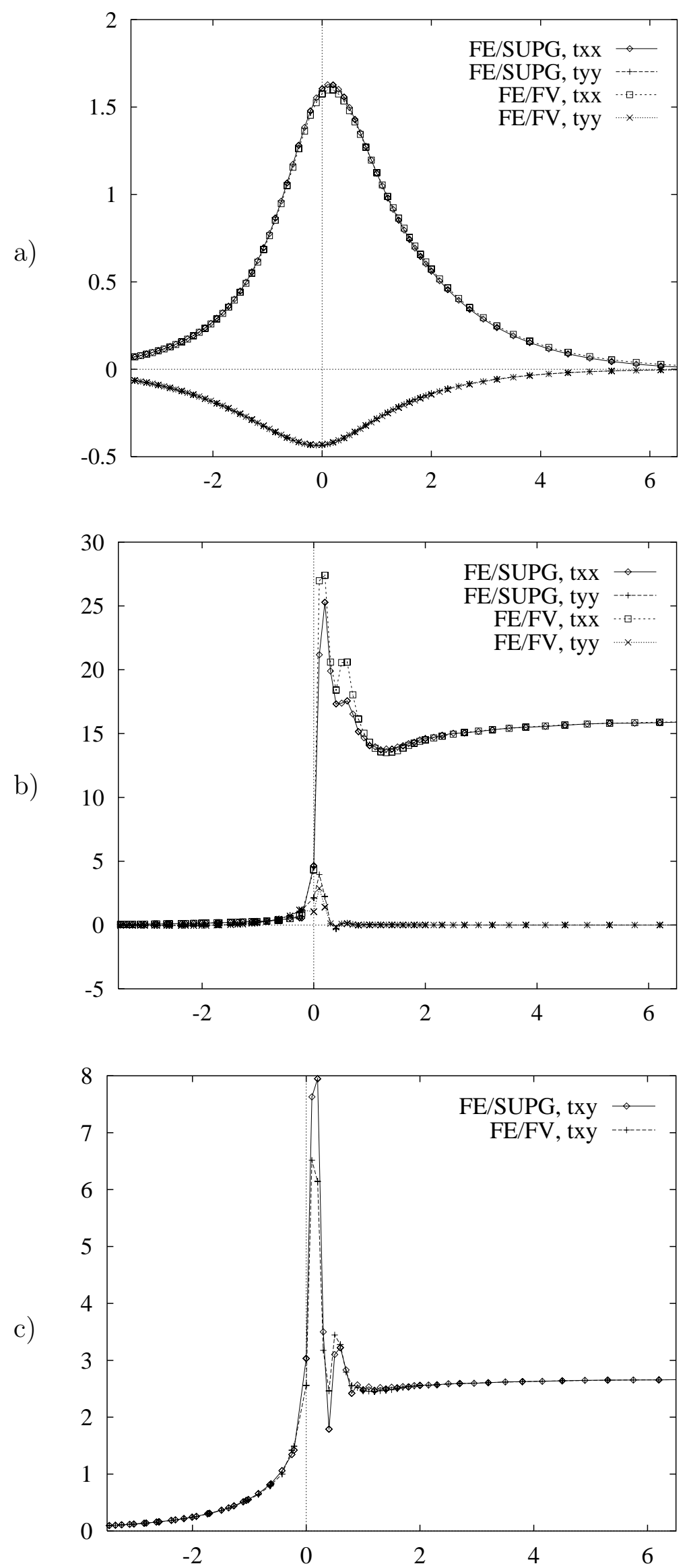

Figure 10: Line plot of stress at centre line a) $\tau_{x x}$ and $\tau_{y y}$ and horizontal line through reentrant corner b) $\tau_{x x}$ and $\tau_{y y}$, c) $\tau_{x y}$; 4:1 contraction flow, FE/SUPG and hybrid FE/FV, $W e=1$. 
FE/SUPG

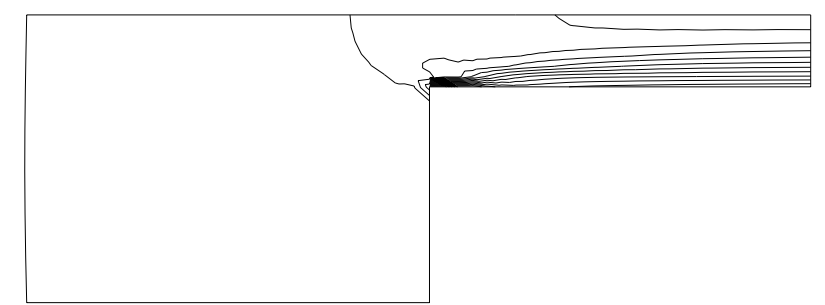

a)

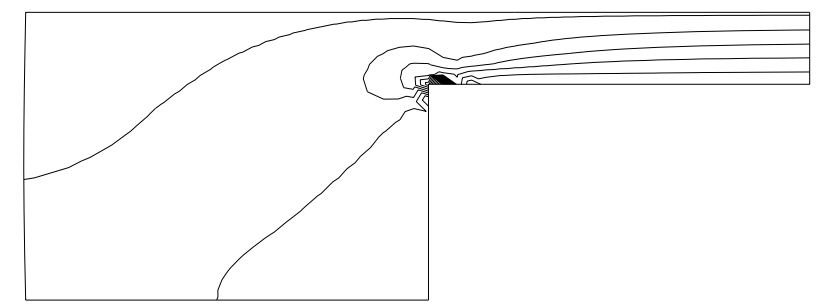

b)

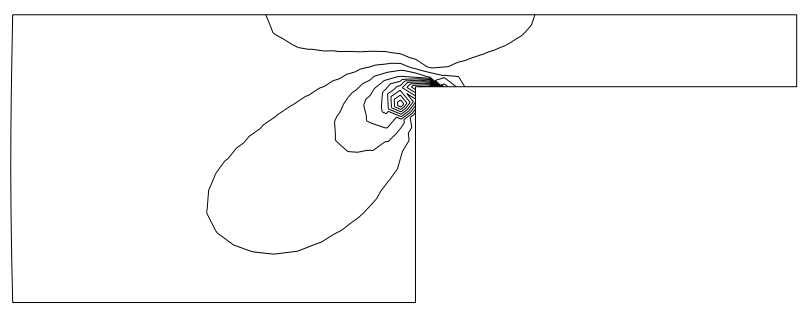

c)
$\mathrm{FE} / \mathrm{FV}$

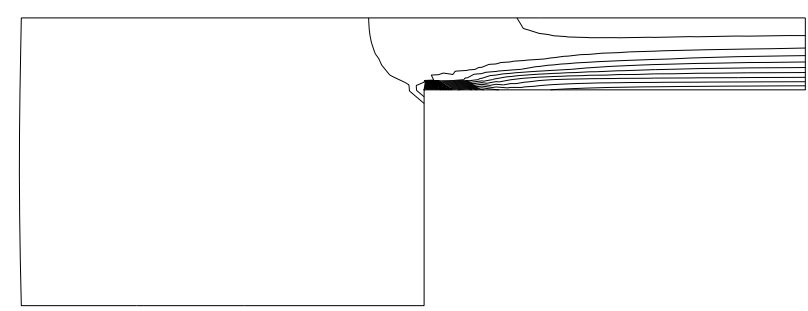

d)

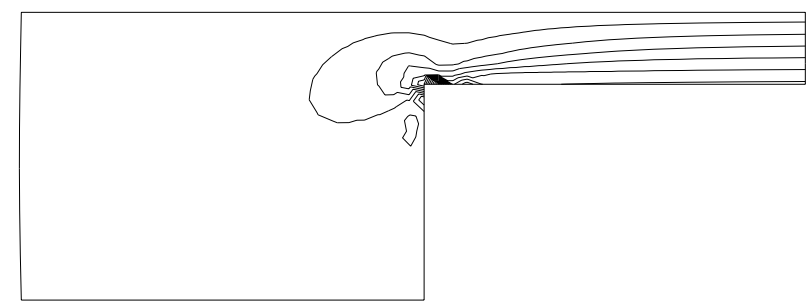

e)

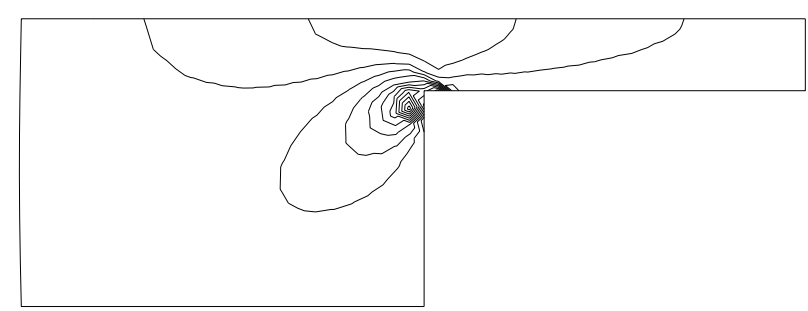

f)

Figure 11: Contour lines of stress, normal and shear, 4:1 contraction flow, $W e=1$, FE/SUPG and hybrid FE/FV a), d) $\tau_{x x}:-0.51$ to 9.21, b), e) $\tau_{x y}:-0.04$ to 9.25 c), f) $\tau_{y y}:-0.31$ to 17.85 . 

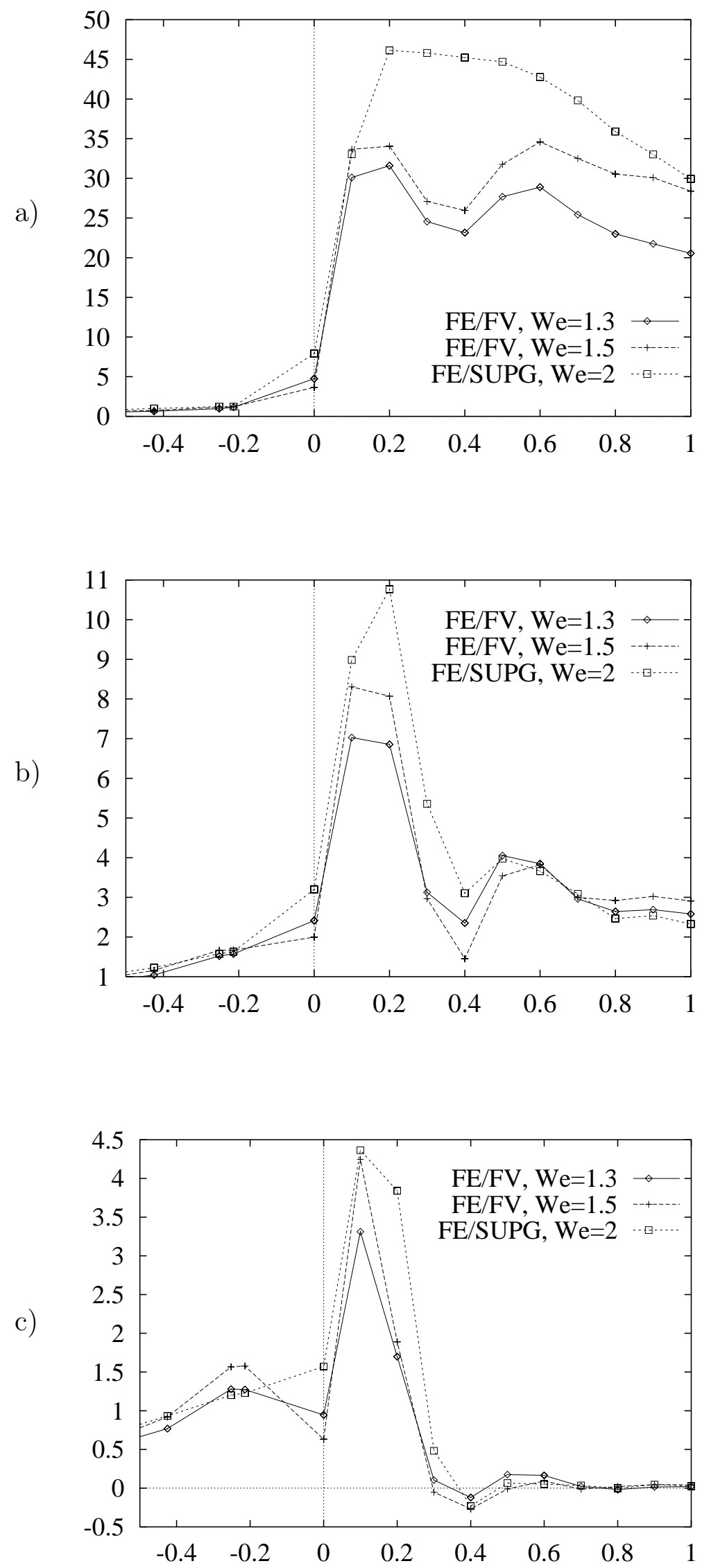

Figure 12: Line plot of stress at horizontal line through re-entrant corner a) $\tau_{x x}$, b) $\tau_{x y}$, and c) $\tau_{y y} ; 4: 1$ contraction flow, FE/SUPG and hybrid FE/FV, various Weissenberg numbers. 
FE/SUPG

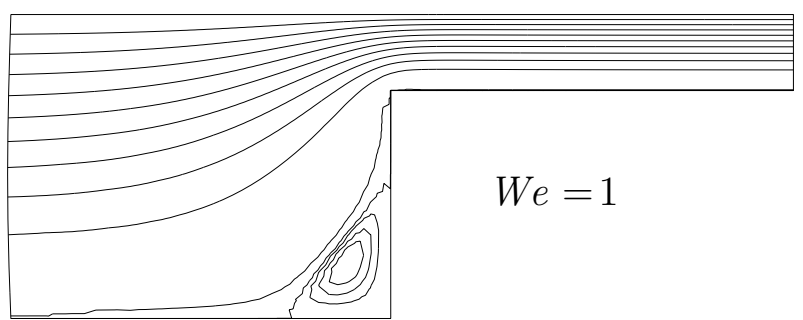

a)

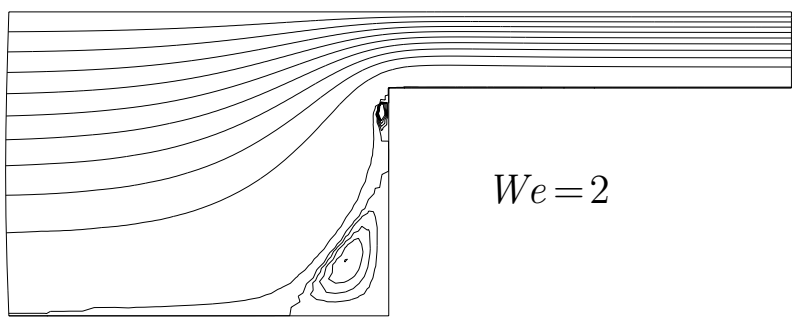

b)

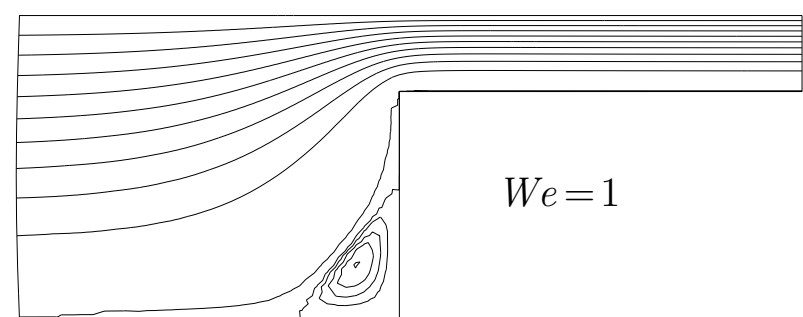

c)

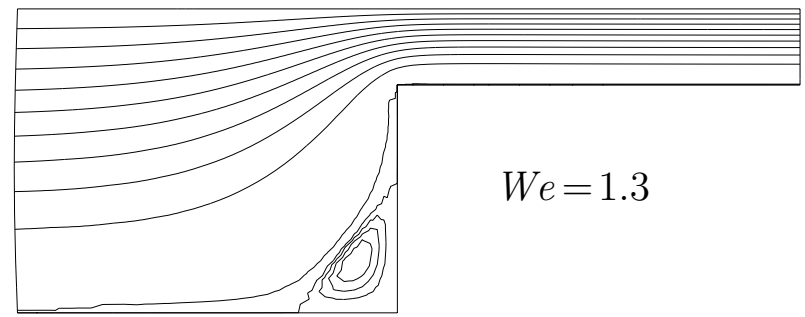

d)

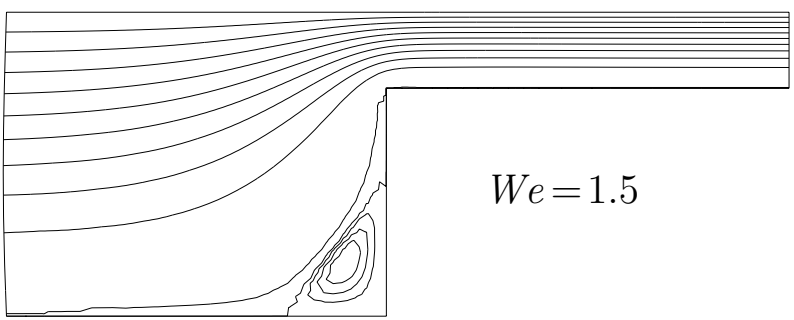

e)

Figure 13: Streamlines for 4:1 contraction flow, FE/SUPG, a) $W e=1$, b) $W e=2$, and hybrid $\mathrm{FE} / \mathrm{FV}$, c) $W e=1, \mathrm{~d}) W e=1.3$, e) $W e=1.5$. 